\title{
Operational research techniques in medical treatment and diagnosis: A review
}

\author{
F. Bartolozzi ${ }^{\text {a }}$, A. de Gaetano ${ }^{\text {b }}$, E. Di Lena ${ }^{\text {a }}$, S. Marino ${ }^{\text {a }}$, L. Nieddu ${ }^{\text {a }}$, G. Patrizi ${ }^{\text {a,* }}$ \\ a Dipartimento di Statistica, Probabilità e Statistiche Applicate, Università degli Studi "La Sapienza", Piazza Aldo Moro 5, \\ 00185 Roma, Italy \\ ${ }^{\mathrm{b}}$ Centro di Studio, Consiglio Nazionale della Ricerche, Roma, Italy \\ Received 1 December 1998; accepted 31 December 1998
}

\begin{abstract}
Many modern techniques for the diagnosis of pathological states in humans and for their subsequent treatment can be posed as nonlinear identification problems of essentially nonlinear dynamic systems or as nonlinear optimal control problems. It can be shown that the linearised versions of such models are inadequate and do not represent at all well the complexity of the problem. Thus, nonlinear estimation and control techniques are required for progress to be made in this field.

The aim of this review is to examine some models suggested in the medical literature for the modelling of certain medical treatments and diagnoses. Then examine how these models can be enriched by using Operational Research techniques so that a better control is provided on the diagnosis and the treatment, as well as the formulation of more precise models of the phenomenon.

The review will present some applications both therapeutic and diagnostic that have appeared in the literature. Special interest will be bestowed on hyperthermic systems in oncological treatment and glucose-insulin dynamics for diabetic patients, while heart dynamics and magnetic resonance imaging will also receive attention. These applications are good examples to show the advantages of Operational Research methods in this field of endeavour.

The outline of the paper is the following. After the introduction, in section two a brief description of nonlinear system models of phenomena will be given, for definitional and descriptive purposes. In section three a discussion of how to apply System theory in the medical field will be presented, together with an analysis of the possible benefits. In section four some applications of dynamical models to medical diagnosis and treatment will be described, while in section five the appropriate conclusions will be stated. (c) 2000 Published by Elsevier Science B.V. All rights reserved.
\end{abstract}

Keywords: Dynamic systems; Optimal control; Medical modelling; Simultaneous identification and optimization

\footnotetext{
${ }^{*}$ Corresponding author. Tel.: +39 649910 735; fax: +3906 4959241.

E-mail address: patrizi@cam07a.sta.uniroma1.it (G. Patrizi).
} 


\section{Introduction}

Operational Research has enjoyed a long tradition of dynamic modelling of phenomena since its inception. Familiar techniques have dealt with difference and differential equations, dynamic programming and optimal control theory. Through the development of variational inequalities and the study of applications to equilibrium transport systems [34], dynamic variables have been introduced and methods have been created to solve systems with dynamic aspects.

The modelling approach in Medicine is essentially of comparative statics, in which the state of the pathology is measured or categorised in suitable classes. Instead, many types of medical treatments and diagnoses would lend themselves well to representing them by suitable dynamic systems, so that not only the state but the change of states are monitored explicitly. Instead in many cases these dynamic aspects are assessed by, what is called in clinical practice, the 'clinical eye', which represents the accumulated experience of the physician in curing such pathologies.

Thus the aim of this review is to examine the principal models suggested in the medical literature for the modelling of certain medical treatments and diagnoses. Analyse how these models can be made more realistic or accurate, through Operational Research techniques and study some recent results in medical diagnosis and treatment which have directly included these Operational Research techniques. The main emphasis will be on the examination of how the newer dynamical methods of Operational Research can be transferred to medical treatment and diagnosis and if this is a useful aspect of research.

To propose a dynamic model of a phenomenon, the traditional method requires that first a suitable model of the process is specified (the form of the relations which define the system), then it must be identified in the parameters, so that the residuals of the estimation obey certain statistical conditions. The estimation is carried out by solving an unconstrained optimisation problem, a special case of which is the famous least-squares algorithm. Lastly, the now quantified model is optimised with respect to a merit function and the defined control variables subject to a series of constraints that will limit the variability of the process.

The theory of estimation is well founded and complete in the case of models linear in the parameters, except that the handling of the statistical conditions on the residuals is a trial and error process. Instead the theory of estimation for functions nonlinear in the parameters is, as yet, incomplete and only partially formalised, rendering it a heuristic process.

The key to modern developments in dynamic modelling, which are very often nonlinear models in the parameters of the process, is to solve a constrained optimisation problem, instead of the unconstrained estimation optimisation, so that the statistical conditions to be satisfied are introduced as constraints in this formulation. It follows that all optimal solutions to the constrained optimisation problems, provide statistically correct estimates.

Further, by defining a more general constrained optimisation problem, the optimisation for estimation and for control can be defined as a single optimisation problem, so that the best control is found compatible with a correctly identified model, which in the nonlinear case will reduce the suboptimisation inherent in the traditional process.

This new simultaneous identification and control algorithm or the new identification algorithm, if there is no control to be exercised, are eminently suitable for the identification of nonlinear dynamic processes, which cannot be solved by traditional methods without incurring in severe suboptimisation and biased estimation.

The analysis of phenomena by a set of dated relations seems to be such a fundamental approach to the understanding and to the prediction of phenomena that many tentative paradigms have been formulated. Thus Systems Analysis [11], Systems Engineering [114], General Systems Theory [107,110], Living Systems Theory [66], and Mathematical System Theory [54] have all sprung from the Laplacian ideas of differential equations systems, with different degrees of formalisation and the application of the scientific method. Here 
we do not propose to examine any of the above approaches, save the last one, which is an axiomitised formal mathematical theory and therefore considered to be suitable as a paradigm for scientific applications.

The outline of the paper is the following. After the introduction, in Section 2 a brief description of nonlinear system models of phenomena will be given, for definitional and descriptive purposes. Here the available identification and control methods will be described, as well as the new methods which are suggested. In Section 3 a discussion of how to apply System theory in the medical field will be presented, together with an analysis of the possible benefits. In Section 4, applications of dynamical models to diagnostic and treatment problems will be described, dealing with the glucose-insulin kinetics; magnetic resonance imaging segmentation, useful in many harder diagnostic problems; the analysis of the dynamics of the heart and finally, the dynamic optimisation of hyperthermic treatment in oncology. In Section 5 the appropriate conclusions will be reached.

\section{Dynamic system modelling}

The representation of phenomena by dynamic systems is more general than their representation by a static system, since the latter will always constitute a special case of the former where warranted. On the other hand, for a given phenomenon its transient may determine the stationary equilibrium, if at all, that will eventually prevail, thus indicating that the dynamic variables may be very important in modelling, even if the stationary state conditions are the desired representation that is sought [90].

Modelling a phenomenon by a dynamic system means imposing the structure of the former on the system variables. It also means that the dated variables, which represent the phenomenon, must agree with the dated estimated values of the system and the mathematical properties of the system variables must apply to the variables of the phenomenon. In dynamic modelling, this requirement is not just that the variable in the two systems be defined compatibly (both integer variables etc.), but their compatibility must extend to their dynamic structure. In short, both must possess compatible properties in their controllability, observability and stability, which will be discussed below. The importance of this is obvious. If a stable phenomenon is modelled by an unstable system, which explodes, the realisation may agree over certain limited intervals, but it is bound to diverge eventually. In particular the properties of the latter cannot be used to represent the former, since they are different. There are many examples of proposed general dynamical systems, which are extremely appealing, but which become very special if examined in their dynamic structure: typically the model will explode or collapse, while the phenomenon thrives $[45,46]$. Thus the aim of this paragraph is to review briefly the main properties of dynamical systems and their principal definitions, so that their structural properties may be considered when identifying and applying such systems.

In Section 2.1 the current dynamical system theory models and their properties will be presented. Dynamic models are usually nonlinear models of phenomena, so it is extremely important to identify models using powerful estimation techniques. Thus in Section 2.2, traditional methods of identification will be recalled and shown to be limited and, consequently, powerful techniques will be presented in Section 2.3. Lastly, in Section 2.4 methods to optimise the dynamical system will be described, as well as a new method which achieves the simultaneous identification and optimisation of a dynamical system, thereby avoiding its possible suboptimisation.

Dynamical systems are generalisations of systems of differential and/or difference equations [54]. So, their solution, where warranted, can be obtained by classical mathematical techniques [28], by Calculus of Variations techniques [84], by Optimal Control techniques [87,39] and by Dynamic Programming [12,2,32]. Computational methods have also been proposed to actually solve systems of differential or difference 
equations, which comprise discretisation techniques and their solution by nonlinear gradient methods and mathematical programming techniques $[33,22,44,41]$.

\subsection{Dynamical systems and their properties}

Mathematical System Theory deals essentially with the study of the dynamical relationships of system under various conditions, more general than those which define difference and differential equation systems [109]. A Dynamical System is a precise mathematical object, so that the study of system theory is largely, although not entirely a branch of mathematics [54,53]. Although very general, mathematical system theory is not neutral with respect to the modelling process. For instance, phenomena may be modelled by dynamical systems if they can be represented as nonanticipatory and the functional forms that define the system are sufficiently smooth [54]. The smoothness requirement can be removed, leading to the theory and methods of discrete systems, which will not be pursued in this review, except in so far as discretisation of the input and output functions will be carried out, for computational reasons [17,22]. Dynamical Systems have been defined at a high level of generality, to refine concepts and perceive unity in a diversity of applications. To obtain interesting results, however, it is necessary to restrict our attention to less general classes of systems.

The fundamental definition of a dynamic system is the following:

Definition 2.1. A Dynamical System is a composite mathematical object defined by the following axioms:

1. There is a given time set $T$, a state set $X$, a set of input values $U$, a set of acceptable input functions $\Omega=\omega: \Omega \rightarrow U$, a set of output values $Y$ and a set of output functions $\Gamma=\gamma: \Gamma \rightarrow Y$.

2. (Direction of time). $T$ is an ordered subset of the reals.

3. The input space $\Omega$ satisfies the following conditions.

(a) (Nontriviality). $\Omega$ is nonempty.

(b) (Concatenation of inputs) An input segment $\omega_{\left(t_{1}, t_{2}\right]}, \omega \in \Omega$ restricted to $\left(t_{1}, t_{2}\right] \cap T$. If $\omega, \omega^{\prime} \in \Omega$ and $t_{1}<t_{2}<t_{3}$ there is an $\omega^{\prime \prime} \in \Omega$ such that $\omega_{\left(t_{1}, t_{2}\right]}^{\prime \prime}=\omega_{\left(t_{1}, t_{2}\right]}$ and $\omega_{\left(t_{2}, t_{3}\right]}^{\prime \prime}=\omega_{\left(t_{2}, t_{3}\right]}^{\prime}$.

4. There is a state transition function $\varphi: T \times T \times X \times \Omega \rightarrow X$ whose value is the state $x(t)=\varphi(t ; \tau, x, \omega) \in$ $X$ resulting at time $t \in T$ from the initial state $x=x(\tau) \in X$ at the initial time $\tau \in T$ under the action of the input $\omega \in \Omega$. $\varphi$ has the following properties:

(a) (Direction of time). $\varphi$ is defined for all $t \geq \tau$, but not necessarily for all $t<\tau$.

(b) (Consistency). $\varphi(t ; t, x, \omega)=x$ for all $t \in T$, all $x \in X$ and all $\omega \in \Omega$.

(c) (Composition property). For any $t_{1}<t_{2}<t_{3}$ there results:

$$
\varphi\left(t_{3} ; t_{1}, x, \omega\right)=\varphi\left(t_{3} ; t_{2}, \varphi\left(t_{2} ; t_{1}, x, \omega\right), \omega\right)
$$

for all $x \in X$ and all $\omega \in \Omega$.

(d) (Causality). If $\omega, \omega^{\prime} \in \Omega$ and $\omega_{(\tau, t]}=\omega_{(\tau, t]}^{\prime}$ then $\varphi(t ; \tau, x, \omega)=\varphi\left(t ; \tau, x, \omega^{\prime}\right)$.

5. There is a given readout map $\eta: T \times X \rightarrow Y$ which defines the output $y(t)=\eta(t, x(t))$. The map $(\tau, t] \rightarrow Y$ given by $\sigma \mapsto \eta(\sigma, \varphi(\sigma, \tau, x, \omega)), \sigma \in(\tau, t]$, is an output segment, that is the restriction $\gamma_{(\tau, t]}$ of some $\gamma \in \Gamma$ to $(\tau, t]$.

A picturesque language is often used to refer to some of the terms in Definition 2.1. For instance the ordered pair $(\tau, x)$ with $\tau \in T$ and $x \in X$ is an event or phase of the dynamical system. The state transition function $\varphi$ (or its graph in the event space) is called trajectory, motion, orbit or solution (of the ordinary differential equation). The input or control $\omega$ moves, transfers, carries, transforms the state $x$, or the event $(\tau, x)$ to the state $\varphi(t ; \tau, x, \omega)$ or to the event $(t, \varphi(t ; \tau, x, \omega))$. The motion of a system is a generic reference to 
$\varphi$. A dynamical system is said to be reversible if the transition function is defined for all values of $t$ and $\tau$, not merely $t \geqslant \tau$.

This notion of a dynamical system is far too general to model specific phenomena. A certain degree of additional structure will have to be imposed, so that the results obtain specificity. However too much structure will deprive the modellisation of much of its intrinsic interest. For instance, if the definition is restricted to finite dimensional time invariant and linear system, the resulting structure starts to look very much like linear algebra. In particular the dynamics of the system become trivial [76].

An other way to model phenomena is through opportune dynamical systems in the input/output sense.

Definition 2.2. A Dynamical System in an input/output sense is a composite mathematical object defined as follows:

1. There are given sets $T, U, \Omega, Y$ and $\Gamma$ satisfying all the properties required by Definition 2.1 .

2. There is a set $A$ indexing a family of functions

$$
\mathscr{F}=\left\{f_{\alpha}: T \times \Omega \rightarrow Y, \alpha \in A\right\},
$$

each member of $\mathscr{F}$ is written explicitly as $f_{\alpha}(t, \omega)=y(t)$ which is the output resulting at time $t$ from the input $\omega$ under the experiment $\alpha$. Each $f_{\alpha}$ is called an input/output function and has the following properties:

(a) (Direction of time). There is a map $t: A \rightarrow T$ such that $f_{\alpha}(t, \omega)$ is defined for all $t \geqslant \imath(\alpha)$.

(b) (Causality). Let $\tau, t \in T$ and $\tau<t$. If $\omega, \omega^{\prime} \in \Omega$ and $\omega_{(\tau, t]}=\omega_{(\tau, t]}^{\prime}$, then $f_{\alpha}(t, \omega)=f_{\alpha}\left(t, \omega^{\prime}\right)$ for all $\alpha$ such that $\tau=l(\alpha)$.

According to this definition a dynamical system may be considered as an abstract summary of experimental data. The experiments are labelled by the abstract parameter $\alpha$ consisting of applying an input and observing the resulting output. Of course these should be conducted under the methodology of the scientific method and different conditions must not be imposed which may implicitly constrain the results of an experiment before it is performed. Condition 2(a) indicates the time at which an experiment is started, while condition 2(b) requires that two experiments be labelled differently, if they yield different results under seemingly identical circumstances.

While the input/output approach may determine a family of functions, the state space approach represents the trajectories in the way indicated, through a unique function, so the latter approach is intuitively more appealing, especially in applications, as it will be shown below. From a methodological approach, the representations are equivalent. It is easy to transform a given system from a state space formulation to a input/output formulation and vice versa [3,54], so it will not be dealt with here.

The problem of Realisation is simply that of constructing a dynamical system in the sense of Definition 2.1 from data provided by Definition 2.2. Thus the System theory approach is just a formal way of looking at the problem of scientific model building. Obviously, for this to work, it is required that the experimental data available is sufficiently rich and plentiful as to permit the definition of the state space, which will tie together, in a certain sense, all the experiments that have been performed. Thus it should not be forgotten that the existence of a state space is not a philosophical problem, but purely a computational one, given a certain desired precision. Some authors insist that certain 'self-consistency' conditions are required for a set of experiments to qualify as a dynamical system [115]. These conditions are unnecessary if the approach described here is adopted, since the state space is obtained constructively and will not depend on certain a priori conditions.

Of course, by imposing suitable smoothness conditions on our system, the system defined here can be represented as a system of differential equations and solved by standard techniques. To this end: 
Definition 2.3. A Dynamical System is smooth if and only if:

1. $T=\mathbf{R}$ the real numbers (with the usual topology).

2. $X$ and $\Omega$ are topological spaces.

3. The transition map $\varphi$ has the property that

$(\tau, x, \omega) \mapsto \varphi(. ; \tau, x, \omega)$

defines a continuous map $T \times X \times \Omega \rightarrow \mathbf{C}^{\mathbf{1}}(T \rightarrow X)$, where $\mathbf{C}^{\mathbf{1}}(T \rightarrow X)$ denotes the family of functions that are once continuously differentiable.

It is now possible to indicate when a Dynamical System defined above can be solved as a system of differential equations, by making use of well known classical techniques [27].

Theorem 2.1 [54]. Let a Dynamic System, in the sense of Definition 2.1 which is smooth, in the sense of Definition 2.3, possess these further characteristics:

1. $T=\mathbf{R}, X$ and $U$ are normed spaces,

2. $\Omega$ is the normed space of continuous functions $T \rightarrow U$ with $\|\omega\|=\sup _{t \in T}\|u(t)\|$

3. $\varphi(. ; \tau, x, \omega) \in \mathbf{C}^{\mathbf{1}}(T \rightarrow X)$ for each $\tau, x$ and $\omega$ and the map $T \times X \times \Omega \rightarrow X$ given by $(\tau, x, \omega) \mapsto \dot{\varphi}(t ; \tau, x, \omega)$ is continuous for each $t$, with respect to the product topology.

Then the transition function $\varphi$ of the smooth dynamic system is a solution of the differential equation

$$
\frac{\mathrm{d} x}{\mathrm{~d} t}=f\left(t, x, \pi_{t} \omega\right)
$$

where the operator $\pi_{t}$ is a map $\Omega \rightarrow U$ given by $\omega \mapsto u(t)=\omega(t)$.

It cannot be assumed generally that the Dynamical System satisfies the conditions of smoothness, nor that it will meet the necessary and sufficient conditions for an optimal control to exist. Thus in general, the Dynamical Systems to be dealt with may have an awkward structure and possess few of these convenient properties.

In this case, two aspects must be distinguished: the definition of the problem by an approximate problem, which will have a solution and the method of solution of the problem that is generated. Thus the optimisation algorithm formulated to solve the approximation problem must be general and robust enough to solve it.

Concerning the first aspect, a suitable discretisation, which will always be problem dependent can be defined for a dynamical system, given above. Thus the discrete dynamical system is:

$$
\begin{aligned}
& x(k+1)=f(x(k), u(k), k), \\
& y(k+1)=g(x(k), k),
\end{aligned}
$$

where the symbols are as before and $T$ has been discretised in periods, indexed by $k=1,2, \ldots$. Specific other assumptions can be introduced as required, as to the form of the input space, the state space and so on.

The dynamical structure of the system will depend on the functional forms of the maps and the set of admissible inputs. Hence a number of interesting problems arise. For instance, given that a system is in a specific state in this period, the problem of determining what states that can be reached in some future period $K$ is a problem of reachability. Also, whether a certain state can be reached in a finite number of periods is a problem of controllability of the system. Here we are concerned in determining a feasible trajectory of the system, with a particular end state. Instead, the determination of the initial state of a system, given that it is at present in a specific state is a problem of observability. From the state at the 
present time and the history of the outputs of the system, it is desired to discover what was the initial state. Briefly it is desired to determine from where the system sprung.

Any model of a real process is a mathematical idealisation containing a number of approximations to reality. In assessing the relative merits of a given model, it is important to determine how the behaviour of the model changes under various types of perturbations in its defining structure, both for the planning of the trajectories, but also to ensure that the phenomenon and its modellisation are sufficiently close and thus will behave throughout the space in a similar way. In an exactly analogous way, it must be verified that the phenomenon and the system model, in the resulting input/output trajectories express similar extensions of reachability, controllability and observability. Otherwise, although very similar at the present time, they will end up with very different properties at some future time.

These concepts are fairly trivial, when discussing linear systems [23], and it has been shown [76] that linear, smooth, finite state constant systems have such a simple structure and their controllability, reachability and observability properties are so simple, as to be reversible in time and not to require any optimal control. Instead the properties of nonlinear systems are extremely intricate and complex and their nonlinear dynamics are worthwhile to study $[24,4,67]$. Moreover their properties are rich enough to permit the modellisation of many phenomena.

Controllability, Reachability, Observability and Stability play also a fundamental role in the identification and in the optimal control of the system model, to which we now turn.

\subsection{Dynamic system identification}

Given an input/output realisation the first step in the modellisation process is to determine the functional form and the parameters of the maps used to represent the phenomenon. The identification process, as it is called, can estimate the system either in state space form or in input/output form. As it has been indicated in the previous section, since these two representations are equivalent, there is no difficulty in identifying the system in one form and then transforming it into the other [3].

System identification is the field of determining the model from experimental data, so that a suitable representation of the phenomenon, at a given level of precision is obtained. While in Engineering and Physics the data can be generated as desired to form a given set of inputs, which is constructed to make the estimation of parameters easier, in other fields, the realisation is already formed and what is available must be used [94].

The given input-output realisation is considered and a functional form is selected. This will require a number of parameters to be determined, by applying one of the many identification methods. After the estimation, the quantified model is compared to the real process, by observing the discrepancies between the actual and calculated values, while cross validation may be carried out by comparing a realisation that has played no role in the estimation to the model output, on applying the inputs of this latter realisation to the estimated model. Suitable tests of goodness of fit may be applied and various distributional assumptions on the residuals checked. In fact, these methods are part of the statical estimation methods, often used.

If the model is not a suitable representation of the phenomenon, then a new model must be suggested and the procedure must be repeated. As in statistical estimation, for the specification of the functional form, statistical tests are carried out on the residuals and the result of the test of hypotheses indicates if this specification is acceptable. However, since the realisation is finite, errors of type 1 (refusing the correct estimates) and type 2 (accepting a false estimate) may be committed, so that a specification may be considered suitable, while in fact it is not, but appears so, only by chance.

To carry out the statistical tests, under the traditional estimation methods, require very large samples, which gives rise, usually, to an ill-posed problems [102]. However, the estimation theory for models linear in the parameters is well founded and demonstrably correct, when the conditions on the residuals are met. For 
the modelling of phenomena by dynamical systems, however, the data is very often serially correlated and the problem of estimation is much more similar to the problem of estimating a time series model. Moreover, very often a model nonlinear in the parameters must be applied, which destroys the convenient theory and the properties of the linear estimation theory.

The problem of identifying nonlinear systems in a suitable way is considered important and much research has been devoted to it [38]. Research has slowly moved from simultaneous linear systems to nonlinear single equation problems to simultaneous nonlinear systems. For this last category the problems are enormous, because the system to be estimated should have the same controllability, reachability, observability and stability properties as the phenomenon originating it. Hence the estimation of the parameters must satisfy many constraints, at least in principle, which will ensure that the relevant properties are met. This cannot be done with the usual methods of unconstrained optimisation.

It has been shown that the dynamics of a linear constant finite state system, which is very easy to identify, have such simple properties as to render it of little use in applications [76]. In fact, for these systems, time can run in either direction, they are always either stable or unstable and simple conditions can be given for their controllability and reachability. Thus no problems arise for these systems, but they are trivial.

To actually determine the parameters of the system, an unconstrained optimisation problem must be solved, which in the case of a linear model in the parameters has a unique solution, while in the case that the problem is nonlinear in the parameters, there may be many solutions or even none at all. The properties of the residuals for each solution may be different, so a complex problem should be solved many times and the properties of the residuals checked [38].

It would therefore seem preferable to define the optimisation problem as a constrained optimisation problem, where in the constraints are placed all the statistical conditions that the residuals should satisfy, so that any solution of this problem will have residuals that satisfy these properties. Thus a correct estimate will be found every time, if a solution exists and if the optimisation algorithm is sufficiently powerful.

\subsection{Nonlinear estimation with constraints}

For nonlinear estimation, the possibility that the resulting optimisation problem may have many solutions, makes it necessary to solve the problem in such a way that the residuals will satisfy the statistical properties, which are required. Thus the aim of this section is to describe how this problem may be solved.

Briefly, it is suggested to solve, instead of an unconstrained optimisation problem, a constrained optimisation problem, by placing all the required statistical conditions as constraints that the residuals must satisfy.

The model to be fitted can be considered purely as a predictive model of a phenomenon [56], indicated also as an inadequate model [20], or alternatively it is desired to approximate the model with a 'true' underlying relationship which characterises it, comprised of a functional form and a given distributional form of errors. This is indicated as an adequate model [20,93].

By postulating a 'true' underlying model, which is however not observable [63], a number of hypotheses on the error structure and on the regressors can be introduced, so that, in the case that these assumptions were true and the realisation were a probabilistic sample from the underlying population, then the estimated model, at a given level of significance, would be a close approximation to the 'true' relationship [49].

The method adopted in this algorithm is to estimate the statistically correct coefficients of a relation by including the statistical properties that the estimate must satisfy as constraints in a constrained optimisation 
problem. Thus a constrained minimum is found, which yields the best constrained least square estimate for the given specification considered.

By changing the independent variables, better and better estimates can be formulated and, also, better estimates may be obtainable by changing the distributional assumptions on the residuals.

The advantage of the method is that at every iteration, a statistically correct estimate is found, if one exists, for that representation and by considering additional variables or nonlinear combinations of the variables so far included, new estimates are formed, with a reduction in the variance of the residual and therefore a better fit.

Thus, in this approach an optimal selection process for new variables is enacted at each major iteration, as well as additional combinations of the existing ones. At each attempt by construction, a correct statistical estimate will be determined, so that the required variance reduction can be pursued. Here the estimate satisfies, through the constraints, the required statistical properties.

Exactly the same considerations can be applied to lagged terms by defining appropriate constraints which ensure that there will be no autocorrelation in the residuals and no heteroschedasticity.

Although the statistical properties that the estimated model should satisfy are well known $[1,49]$ nevertheless there has been hardly any work done on methods to constrain the parameter space, so that correct estimates can be obtained. Some work has been done in determining the estimates, if there are restrictions on the coefficients, for instance nonnegativity, or linear relations [1], but very few results have appeared to date concerning the introduction of constraints in the parameter space to ensure statistically correct estimates [61].

Consider a realisation of a phenomenon composed of $n$ measurements, in which at each measurement a scalar quantity of some variable is measured, indicated by $y_{i}(i=1,2, \ldots, n)$ and a set of $p$ independent variables or regressors are also measured, indicated by $x_{i}$. It is desired to determine a functional form $f: \mathfrak{R}^{m} \rightarrow \mathfrak{R}$, and the determination of a set of suitable coefficients, $\theta$ such that

$$
y_{i}=f\left(x_{i}, \theta\right)+u_{i}
$$

where $u_{i}$ is a residual, with a null mean, a finite variance and statistically independent.

In the least squares estimation procedure, there is no mechanism to ensure that the derived estimates inherit the properties of the underlying 'true' relationship, so from an improbable sample, absurd results may be obtained or vice versa and there seems no way to ensure that the procedure converges to a correct estimate.

Rather than postulate the existence of an adequate model of the relationship and a series of unverifiable hypotheses on the elements of the relationship, it is preferable to determine the coefficients and the functional form in such a way as to ensure that the residuals satisfy certain conditions so that the estimate obtained, under mild hypotheses, are efficient, unbiased, consistent and the residuals are independent, serially uncorrelated, homoschedastic and asymptotically normal. Naturally, the asymptotic properties concern limit values of the properties of the sample, as the sample size grows.

Thus a randomly drawn realisation is obtained and it is desired to define a mathematical combination of certain elements, called the regressors and indicated by the matrix $X \in \mathfrak{R}^{n \times p}$ (such that when substracted from the vector $y \in \mathfrak{R}^{n}$, a residual vector $u \in \mathfrak{R}^{n}$ is obtained, which it is desired to render as small as possible in the Euclidean norm. To ensure that such an approximation constitutes a regression, a number of conditions are imposed on the process of determining the minimum. Thus we assume that the values of the regressors and of the endogenous variable are bounded, and that the regression function considered in Eq. (1) is continuous in all its arguments. Further, it is assumed that the exogenous variables are meaningful, which is the nonlinear version of the exogenous variables being noncollinear, as is indicated, [63]. 
Formally, the nonlinear regression problem becomes

$$
\begin{array}{ll}
\operatorname{Min} & \sum_{i=1}^{n}\left(y_{i}-f\left(x_{i}, \theta\right)\right)^{2}, \\
\text { s.t. } & g(y, x, \theta) \geqslant 0,
\end{array}
$$

where $g(\cdot)$ is a set of conditions imposed on the residuals in terms of the data and the function and the coefficients to be determined, so that the residuals are unbiased, have minimal finite variance, are serially uncorrelated, homoschedastic, asymptotically consistent and follow a normal distribution.

Each of these properties are imposed as mathematical conditions on the optimisation problem to be solved and so the determination of an optimum solution for the problem (2) ensures the satisfaction of all the conditions imposed and therefore for every specification, the estimation process assures that the conditions are fulfilled, if it is possible. It is then easy to show that the property of asymptotic consistency follows, both when the model is inadequate or when an adequate model is postulated, which satisfies the usual assumptions, as indicated [49].

Consider, therefore the following constrained optimisation problem:

$$
\min S_{n}^{2}(\theta)=n^{-1} \sum_{i}^{n}\left[y_{i}-f\left(x_{i}, \theta\right)\right]^{2},
$$

s.t.

$$
\begin{aligned}
& \sum_{i}^{n}\left[y_{i}-f\left(x_{i}, \theta\right)\right]=0, \\
& \left|\frac{n \sum_{i=2}^{n}\left[y_{i}-f\left(x_{i}, \theta\right)\right]\left[y_{i-1}-f\left(x_{i-1}, \theta\right)\right]}{(n-1) \sum_{i=1}^{n}\left[y_{i}-f\left(x_{i}, \theta\right)\right]^{2}}\right| \leqslant 2 / \sqrt{n}, \\
& \left|\frac{n \sum_{i=3}^{n}\left[y_{i}-f\left(x_{i}, \theta\right)\right]\left[y_{i-2}-f\left(x_{i-2}, \theta\right)\right]}{(n-2) \sum_{i=1}^{n}\left[y_{i}-f\left(x_{i}, \theta\right)\right]^{2}}\right| \leqslant 2 / \sqrt{n}, \\
& \ldots \ldots \ldots \\
& \left|\frac{n \sum_{i=s+1}^{n}\left[y_{i}-f\left(x_{i}, \theta\right)\right]\left[y_{i-s}-f\left(x_{i-s}, \theta\right)\right]}{(n-s) \sum_{i=1}^{n}\left[y_{i}-f\left(x_{i}, \theta\right)\right]^{2}}\right| \leqslant 2 / \sqrt{n}, \\
& \frac{1}{2} g^{\prime} X\left(X^{\prime} X\right)^{-1} X^{\prime} g-\frac{n}{2} \leqslant \chi_{\alpha: k-1}^{2}, \\
& \sum_{i=1}^{n}\left[y_{i}-f\left(x_{i}, \theta\right)\right]^{2 r+1}=0, \quad r=1,2, \ldots, \\
& \sum_{i=1}^{n}\left[y_{i}-f\left(x_{i}, \theta\right)\right]^{2 r}=\frac{(2 r) !}{r ! * 2^{r}}\left(n^{-1} \sum_{i}^{n}\left[y_{i}-f\left(x_{i}, \theta\right)\right]^{2}\right)^{r}, \quad r=1,2, \ldots
\end{aligned}
$$

It is desired to minimise the residuals of a function, by determining the appropriate coefficients, Eq. (3). The functional form can be set a priori, or the constrained optimisation can be defined in a more general way, so that optimisation is carried out, both with respect to the unknown parameters and to the unknown combination of variables. The actual form chosen, as well as the values of the coefficients estimated, will depend on the problem data.

Eq. (4) imposes that the residuals of the random sample have a null sample mean, while Eqs. (5)-(7) indicate that null serial correlations up to a lag of $s$ periods are imposed and must be satisfied. These constraints must not be significantly different from zero, so the statistical test described in [7] is applied. 
This requires that the absolute value of the autocorrelation coefficients be smaller or equal to $2 / \sqrt{n}$, where $n$ is the size of the sample. It is suggested that the lag $s$ is the nearest whole number smaller than $n / 5$.

Eq. (8) requires that the residuals be homoschedastic. It is obtained by regressing the original variables, or a subset or a transformation of these, on the normalised square of the residuals, which are indicated by the vector $g$. In Eq. (8), as it can be seen, all the variables are regressed on the transformed residuals, which is the usual case [18]. The test indicated in Eq. (8) can be applied, where the number of degrees of freedom will be $(k-1)$ where $k$ is the number of regressors in the original problem and $\alpha$ the desired significance level. In the mathematical program, if $k$ the number of regressors can vary, the coefficient must be determined from an automatic table look-up in the program or it must be estimated by a formula for all possible values of $k$ and for the given significance level.

Finally the Eq. (9) indicate that all odd moments considered, from the third to the $2 r+1$, are null, while the even moments, Eq. (10) must have values indicated by statistical theory for the normal distribution, or by the required values for an alternative distribution of the exponential family.

Clearly the optimisation problem above has been defined in a form suitable for a statistical explanation, rather than in its computational form, which would be quite different, for numerical reasons.

It is of course well known that for a constrained optimisation problem, the values of the independent variables, here the values that the coefficients will take on, and the values of the auxiliary variables, must be such as to satisfy all the constraints and since the constraints impose certain properties on the residuals, it follows that these conditions must be satisfied for there to be a solution.

The proof that the statistical properties of the residuals are: unbiased, have minimal finite variance, are serially uncorrelated, homoschedastic, asymptotically efficient and follow a normal distribution is given elsewhere [79].

Since, it cannot be assumed that the global minimum to problems (3)-(10) is unique, no asymptotic consistency properties can be formulated. In fact, this means that alternate parametrisations exist for the model specified and the data, so that two or more models exist, which are statistically just as good. The situation under the traditional nonlinear least square estimation is exactly equivalent, except that in the latter additional assumptions are usually introduced to ensure asymptotic consistency of the estimate. If this assumption is postulated in the case described here, then the estimates of this procedure will be asymptotically consistent as well.

The best way to ensure asymptotic consistency in this procedure, which cannot be imposed in the traditional method is to adopt a lexicographic criterion on the estimates. Thus, the unique estimate is chosen, which is lexicographically minimum with respect to all the alternate optimal estimates.

The required statistical properties of the estimates can be met, by solving a suitable optimisation problem, so it is necessary to indicate that the solution can be found by a suitable algorithm. The algorithm suggested is G.O.Al [77,80,81] where general convergence conditions are given, together with many applications, which are nonconvex in all their parts.

Thus, granted that the algorithm described by Eqs. (3)-(10) if it has a solution, the global minimum solution can be found in a finite number of steps, it is interesting to inquire the relationship that exists between the solutions found by a traditional least squares algorithm and the one proposed here.

The constrained problem will find the same solution as the least squares solution, if the latter satisfies the conditions of [49], proposed in the constraints (4)-(10). However, in general not all these conditions may be satisfied.

If, for the estimation problem there is no point where the residuals, and therefore presumably also the underlying population errors are not identically distributed with mean zero and with a finite variance, then the solution found by the method of least squares will not be a feasible solution to the constrained problem. Moreover, if there exists a solution for a given estimation problem, which meets all the statistical properties required, the proposed algorithm will find such a solution, while the traditional least squares method will not, unless the solution coincides with the unconstrained minimum of the problem. This is especially 
important in nonlinear least squares problems. The problem with the traditional technique is that the possible parameter values may not be appropriately constrained to make possible a correct and adequate estimate. This difficulty can of course arise, in nonlinear statistical estimation, purely because of sampling variability and the presence of extensive nonlinearities, as is often the case.

The sequential nonlinear estimation by a constrained algorithm (Se.N.E.C.A.) [78] is more robust for nonlinear estimation than the traditional implementations and is more flexible in capturing the relationships depicted by the data, guaranteeing that the estimates formulated are statistically correct.

\subsection{Simultaneous estimation and optimisation}

In nonlinear estimation, as it has been seen above, there may be many alternative sets of parameters, which satisfy the data. This is similar to time series analysis, where alternative models can be specified and the parameters estimated, which lead to different models all equivalently good. Thus, if it is desired to obtain a predictive model of the process, to determine the best control, it may be found that different models, which are all equivalent, may give rise to very different control policies. With nonlinear models, the pluricity of solutions may require to determine the optimal control for each solution, so that the best control policy is eventually determined. The way out of this burden would be to define an algorithm which estimates and optimises the model at the same time. This is however considered a very difficult problem, with traditional algorithms.

The guarantee that the properties of the estimates are always correct, allows to simultaneously carry out the estimation and the optimisation of the system, by defining a larger problems in which the parameters and the control variables are considered system variables to be optimised. The aim of this section is to describe the way that this can be enacted.

Consider the following optimal control problem, which has been suitably discretised:

$$
\begin{aligned}
& \text { Min } J=\sum_{1}^{K} c(y(k), u(k), x(k)), \\
& \text { s.t. } \\
& x(k+1)=f(x(k), u(k), w(k)), \\
& y(k+1)=g(x(k), v(k)), \\
& w(k) \in W, \\
& v(k) \in V,
\end{aligned}
$$

where $w(k), v(k)$ are random vectors of residuals, drawn from suitable probability spaces $W, V$.

The problem consists in the determination of the functional form, the estimation of the parameters and the optimisation of the system according to the criterion function, when it must be assumed that they are all interrelated. The functional form can be approximated with an appropriate polynomial function, while to the system (11)-(15) the statistical constraints must be added to determine a solution. Thus two sets of statistical conditions must be added, a set controlling the residual variable $w(k)$ and the other set controlling the residual $v(k)$ above.

The statistical consistency and the mathematical convergence results have been given elsewhere [60,79], together with some applications results, so they will not be given here.

The resulting optimisation system can operate in two modes. In the first mode, off-line, the data is used and an optimal control trajectory is found over the given required period. In this mode, no adaptation is envisaged and the problem is run until the last period. In the second mode, or on-line mode, the optimal control trajectory is found for the whole period, but given an elementary period, the control is revised at the end of each elementary period, by considering the divergence between the actual result of the period and the 
predicted result for the period. It is hoped that, as time passes, the accumulation of more information will require less revisions in the optimal trajectory. Thus an adaptive and learning capability is added to the algorithm, since the optimal policy is revised on a roll out basis, as results become available. Under suitable conditions these revisions become less and less and the optimal policy becomes more and more the surely correct one. However, at any moment a great enough perturbation can occur, which will render the optimal policy no longer optimal, so that at the next revision, the system will adapt and a new policy formulated. This is extremely important in this schema of modelling by suitable nonlinear dynamical systems all those problems which require an adaptive control policy, as in the research here described.

\section{Dynamic modelling in medicine}

Medical diagnoses are used to determine the pathological state of an individual. To determine that a given patient is in a particular morbid state, it is required to dispose of a model of how that pathology manifests itself and what measurements will lead to ascertaining its existence. Pathologies may affect the state of the human system, in which case one or more measurements suffice to determine if a certain pathology affects the patient, or whether one of a series of pathologies affects the patient, or it may affect the control mechanism or the dynamics of the response of the system. Since many factors can influence the measurements that can be taken, the problem of diagnosis is a difficult decision problem in which the accuracy of measurements and the correct choice procedure are essential for good diagnosis. To this end, pattern recognition techniques can be used to advantage [70], which if implemented correctly can provide very precise diagnosis.

However, for many pathologies, there is no stationary morbid state associated with a pathology, which may be recognised by the appropriate set of experiments, but rather the pathology is associated with a system malfunction, which must be diagnosed by comparing the patient to the normal system reactions. To determine these latter kinds of pathologies, a suitable experiment must be performed to measure certain parameters of the dynamic system, by monitoring the dynamic system over a certain interval. Then, by comparing the reaction curves or the system parameters obtained with certain curves or parameters obtained from normal individuals, the diagnosis can be made.

Thus diagnosis is really a problem of observability of the system and treatment gives rise to the problem of controllability and/or reachability. Naturally, if a control is enacted, the problem of stability of the system should be at the forefront of attention. Dynamical System theory provides therefore an experimental axiomatised and formalised scientific procedure for the diagnosis and treatment of pathologies.

For this technique to be useful, the assumed dynamical system, which will be estimated from the data, must fulfil all the properties which the real system manifests. In particular the former must be controllable, observable and stable, if the latter has these properties and the estimated trajectory must coincide with a high accuracy to the real trajectory, as otherwise these two systems must be different and there is no certainty that they differ by some unimportant aspect with regard to the pathology.

Lastly, as an extension of this, it is of some importance that the assumed dynamical system refers to a sufficiently broad set of individuals. If a different dynamic system has to be identified for each individual, then of course, no comparisons and hence diagnoses are possible, since there is no control system, as there is no assurance that sane people have a common dynamical system, while sick people each differ in their response. This just means that the dynamical system has not been defined sufficiently generally.

Three frequent objections are moved by expert clinicians to the use of axiomatised formalised methods in Medicine. The first regards the great variation in the particular aspects of a pathology: no two humans are sick in exactly the same way [95]. Secondly, the use of a closed loop control policy appears to make the day by day therapeutic value of a treatment all but useless, while it is known that this is not so [112]. Thirdly, many patients appear to have all the symptoms of a pathology, but do not have that illness, or 
have very few symptoms, but have the given pathology: this is known as the false positive and false negative problem. The conclusion of these preoccupations is that the phenomena are more complex, as practice indicates, than the aspects that can be modelled by these systems.

Dynamic models can be formulated, by using the algorithms as described here, as complex and as varied as is vouchsafe on the basis of the available experimental data. Various problems have been solved with more than 300 variables and about 900 constraints [80], which is certainly an order of magnitude larger than the relationships that can be gathered by expertise. However, the point is well taken, because it is the model that must be adapted to reality and not the other way round, as is often the case.

Further the daily indications of the expected state, which can be compared through a specific and welldefined series of measurements to the actual state will suggest modifications of therapies and plan the optimal control trajectory with much greater precision and detail. Thus these methods are designed to add objectivity and precision to the therapeutic treatment of the clinic. Scientific method is based on accurate predictions and controlled experiments. This approach does exactly that, as it was described in Section 2.

Again, the lack of specificity as indicated by the existence of false positives and false negatives will appear here as a problem of observability. Given a state and a series of outputs, if this problem arises, it means that the systems were earlier in different states. The axiomatic formalised approach adopted here would not allow any conclusions to be drawn or the system to be estimated. On the other hand, there might not be any contradiction, on more detailed modelling of the phenomenon, in which case, the therapeutic contradictions would have been cleared.

It is not implied, here, that the approach indicated can be a substitute for the expert, but rather it should be considered to be a useful tool to the clinician. In fact, many diagnosis and treatments are based on the evaluation on the part of the clinician of certain aspects, which in some cases, will lead him to discern a particular pathology and in other cases to see the problem in a different way. If we suppose that the symptoms might be exactly the same in the two cases, the resulting interpretation would be unscientific. The expert can of course change his mind, if he is conscious of it, based on a new vision or on new data. This change must be motivated by firm principles and it is essential to be able to determine what would have happened if the diagnosis or treatment had been applied previously. This is why a formal deductive approach, such as this one is essential. It would be only misleading to himself and to the community, if he believed that the diagnosis was exactly in the same line as the previous ones, while it was not.

\section{Applications of dynamical modelling}

The aim of this paragraph is to present four applications as examples of the type of medical problems that would benefit from the dynamic modelling described. This is not meant in any way to be an exhaustive analysis, but the examples which will be illustrated below are considered useful to try to present the results that this approach renders possible.

The four instances which will be analysed consider:

- glucose-insulin kinetics,

- magnetic resonance imaging,

- heart dynamics,

- optimisation in hyperthermic treatments.

\subsection{Glucose-insulin kinetics}

The determination as early as possible whether an individual is diabetic or not is an important problem both for the patient and for society as a whole. It is recognised that an early diagnosis of diabetics, allows 
one to carry out specific therapies, which will postpone or avoid some serious effects later. Thus the diabetic can lead a more normal life and have a much longer life expectancy.

The homeostasis of glucose, involving the secretion of its controlling hormone insulin by the pancreas has been the object of much research, almost all requiring the specification of a suitable dynamic model and the determination of some index to indicate the derangements of the sensitivity of tissues to insulin in diverse pathological conditions like diabetes, obesity and cardiovascular disease. In short, by forming a sufficiently accurate model of the time path of glucose and insulin for suitable groups of subjects of various types, it is hoped to form some synthetic measure of this system, which will indicate the specific pathology suffered by the individual.

There are three main types of procedures to measure the time paths of the glucose and the insulin: the pancreatic suppression test, the euglycemic hyperinsulin clamp test [31], the Intra Venous Glucose Tolerance Test, which is the most popular at the present time [14]. Here, the analysis will be performed with respect to this test only. The test consists of injecting intravenously a bolus of glucose and sampling at regular intervals the glucose and insulin plasma concentrations for a period of about three hours.

Various models have been suggested to interpret this dynamic data as in [13] where seven distinct models of glucose uptake are proposed to account for the glucose kinetics and on the basis of identifiability criteria, the medical significance of parameters and the goodness of fit of the model, a single model is selected, which has since been known in the literature as the 'minimal model'. This will be taken as the basic model of the traditional analysis.

The minimal model is presented in two versions: the first part uses two differential equations in state space form to describe the time course of plasma glucose and insulin concentration, which are only related through the state of the system, [13], while in a later paper the model has been extended with a third equation to account for the time course of pancreatic insulin released in response and independently of the glucose stimulus [99].

Consider the following variables:

$G(t)(\mathrm{mg} / \mathrm{dl})$

$I(t)(\mu \mathrm{UI} / \mathrm{ml})$

$X(t)$

$p, b$ blood glucose concentration at time $t$ ( $\mathrm{min})$, blood insulin concentration, state vector for the representation, or interpretable as an auxiliary function representing insulin-excitable tissue glucose uptake activity, proportional to insulin concentration in a 'distant' compartment, parameters of the system.

$$
\begin{aligned}
& \frac{\mathrm{d} G(t)}{\mathrm{d} t}=p_{1} G(t)-X(t) G(t)+p_{4}, \\
& \frac{\mathrm{d} X(t)}{\mathrm{d} t}=-p_{2} X(t)+p_{3} I(t) .
\end{aligned}
$$

As can be seen, the model originally presented is a state-space model with one state equation and one output equation. The system has a single control variable, given as Insulin, which is itself partially controlled by the glucose level. Thus it should not be considered as an exogenous control variable.

In [99] a third equation was added, to model the insulin endogenous variable, so that the system can be written

$$
\frac{\mathrm{d} G(t)}{\mathrm{d} t}=b_{1} G(t)+X(t) G(t)+b_{1} G(0)
$$




$$
\begin{aligned}
\frac{\mathrm{d} X(t)}{\mathrm{d} t} & =-b_{2} X(t)+b_{3} I(t)-b_{3} I(0), \\
\frac{\mathrm{d} I(t)}{\mathrm{d} t} & =b_{4}\left(G(t)-b_{5}\right)_{+}-b_{6} I(t)+b_{6} I(0), \\
G(0) & =b_{0}, \\
X(0) & =0 \\
I(0) & =b_{7}
\end{aligned}
$$

where ()$_{+}=\max \left(G(t)-b_{5}, 0\right)$.

The fundamental changes in this system are that all the basic variables are measured from their initial time values except the multiplicative term, which is measured in absolute terms. This is notationally untidy. In fact, there is no loss of generality if this product was also measured from the offset. The addition of the third equation, if the resulting system is identifiable could provide a stabilisable control, which is exactly what should happen in the human body. In other words, the level of free insulin at time $t$, depends on the level of glucose present at time $t$, measured from an offset point. In this case, however the state of the system becomes superfluous, since insulin affects the state of the system, through Eq. (18), but the state of the system does not affect the level of insulin, since no such term is present in Eq. (20). Thus the only use of the state variable in Eq. (18) is to provide a parameter, which will be a function of time and the level of insulin and affects the rate of change of the glucose level in the body. The model, as given, does not have any explicit variables for individuals, i.e. whether young, old, healthy or not, because it is assumed that these will alter the coefficients of the system. Thus, each individual responds to a particular model or groups of like individuals respond to one basic model for the whole group. In fact, the authors suggest that an Insulin sensitivity index can be calculated, which depends purely on the parameters of the dynamic system (16)(17). Thus for this system the insulin sensitivity index is $E=-p_{3} / p_{2}$, while for the system (18)-(20) the sensitivity index is given as $E=b_{3} / b_{2}$ [99].

The model has gone through some improvements in the experimental setup, the data analysis, without, however any change in the descriptive equations per se. Thus to implement this dynamic system as a diagnostic instrument, samples of individuals with a given pathology must undergo the bolus test and their glucose and insulin levels are recorded at given times for a certain period after the intravenous injection of the glucose bolus. These readings form a time series and each series for each individual is used to determine the coefficients for the dynamic system. The identified coefficients will vary from individual to individual, so statistical tests should be constructed to ensure that the sensitivity index differs significantly for a pathological case and a healthy one.

Several applications have been reported in the literature to measure the insulin sensitivity index for different patient populations, including diabetes [71,111], aging [25,73], hyperthyroidism [83], hyperparathyroidism [55], myotonic dystrophy [85], pregnancy [19], gynaecological conditions [21], obesity [13], hypertension [19], cirrhosis [58,64], ethnical subpopulation [42,43,50,69,98], siblings of diabetic patients, $[29,51,88]$ and during pharmacological tests [62].

With such an important body of research all tied to a given model, it becomes extremely important to ensure that at the required level of precision, the model is accurate. Suppose that the insulin sensitivity index is affected in a systematic way by various factors and that moreover insulin and glucose do not enter linearly in the given relations but the system is nonlinear. Then, for a given patient, his insulin sensitivity index will differ depending on these factors and in fact the asymptotic value may not exist.

Secondly, the distribution of the insulin sensitivity index across patients must cluster in significantly different groups, for otherwise it would not be possible to infer from the value of the index, the pathology suffered. Thus a multiplicity of effects may be discernible, but the specific factor may not be identifiable, in the general case. 
Thirdly, the representation of the glucose-insulin kinetics by a first order system, with a nonlinearity induced by the parameter affecting the rate of change of the glucose level implies that certain properties of this particular dynamic system must be verifiable in the actual system. In particular, if the mathematical system shows that for certain values the system is unstable and gives rise to an ever increasing rate of change in glucose and insulin, then this must occur in reality and patients must be known to suffer such distress.

A careful analysis [35] has shown that there are many problems connected with these models and that in fact, all three objections are relevant. Thus the results cited, obtained in the literature could be improved upon or extended, if more accurate relationships were posited and then the system identified.

So a data set composed of 29 individuals was considered, belonging to three groups: healthy-young (8), healthy-aged (7) and cardiac-aged (14), where the number in parentheses indicates the members of the group. Each subject received a bolus of glucose and the concentration of glucose and insulin was recorded at intervals, with 21 valid readings per individual, starting at 8 minutes after the bolus was applied. The first four readings, at $0,2,4,6$ minutes were used to start the dynamic process To this data the algorithm Se.N.E.C.A. [78] was applied, with the indication that the data was subject to replication in three groups and that the model could be dynamic and expressible as a ratio polynomial. The best model identified was in fact a ratio polynomial indicated by the following equations.

Let:

$A(0)$ a dummy variable that is set to 0 or 1 depending whether the individual is young or aged, $A(1)$ a dummy variable that is set to 0 or 1 depending whether the individual is healthy or cardiac.

$$
\begin{aligned}
& G(t)= \\
& \frac{34.3025-0.3302 I(t)-5.4984 A(0)-0.7428 G(t-1)+0.0584 I(t-1)+0.0188 I(t-2)-0.00238 G(t-1) I(t-1)}{(1-0.0038 I(t)-0.0003 I(t) A(0)-0.0002 I(t) A(1))}, \\
& I(t)= \\
& \frac{16.0599-0.1487 G(t)+0.0404 G(t-4)+0.6201 I(t-1)+0.0307 I(t-3)-0.0033 G(t) I(t-1)}{(1-0.0049 G(t)-0.0005 G(t-1)+0.0001 G(t) A(0)-0.0002 G(t) A(1))} .
\end{aligned}
$$

Apart from the discretisation that has to be introduced for computational reasons, the model in this version is much more complex. The glucose at any period depends on the glucose at the previous period and enjoys a multiplicative reduction effect proportional to the value of the product of the glucose and insulin of the previous period. Moreover it depends on the insulin levels both at that period and at the previous two periods, forming thus an autoregressive time series of the insulin. On the other hand the level of insulin depends on the value of glucose at that period and at four periods before while again there is an autoregressive effect on the insulin and a correction given by the product at the present period of the glucose and at the previous period of the insulin.

A full analysis of this relationship and a complete comparison with the minimal model is given in [37], so it will not be repeated here. Instead, for this model in Fig. 1 the glucose curve for each individual are given and the calculated values of the glucose curves are drawn for each individual based on the model (24) indicated above, with the appropriate values for the parameters $A(0)$ and $A(1)$. The analysis of the stability of this nonlinear system is more complex than in the linear case. The given system is locally stable around the initial points and is instable for possible extreme values of the sane individuals, although such values are not supposed to occur in these individuals.

In Fig. 1 for the glucose levels and in Fig. 2 for the insulin levels, the available data and the fitted equations are given for all the individuals, which are run one after the other. Each graph is for one group, except for the cardiac-aged group that has been divided into two subgroups. The fit, as can be seen, is very good and the model is more realistic, since lagged values of insulin in the glucose equation and lagged 

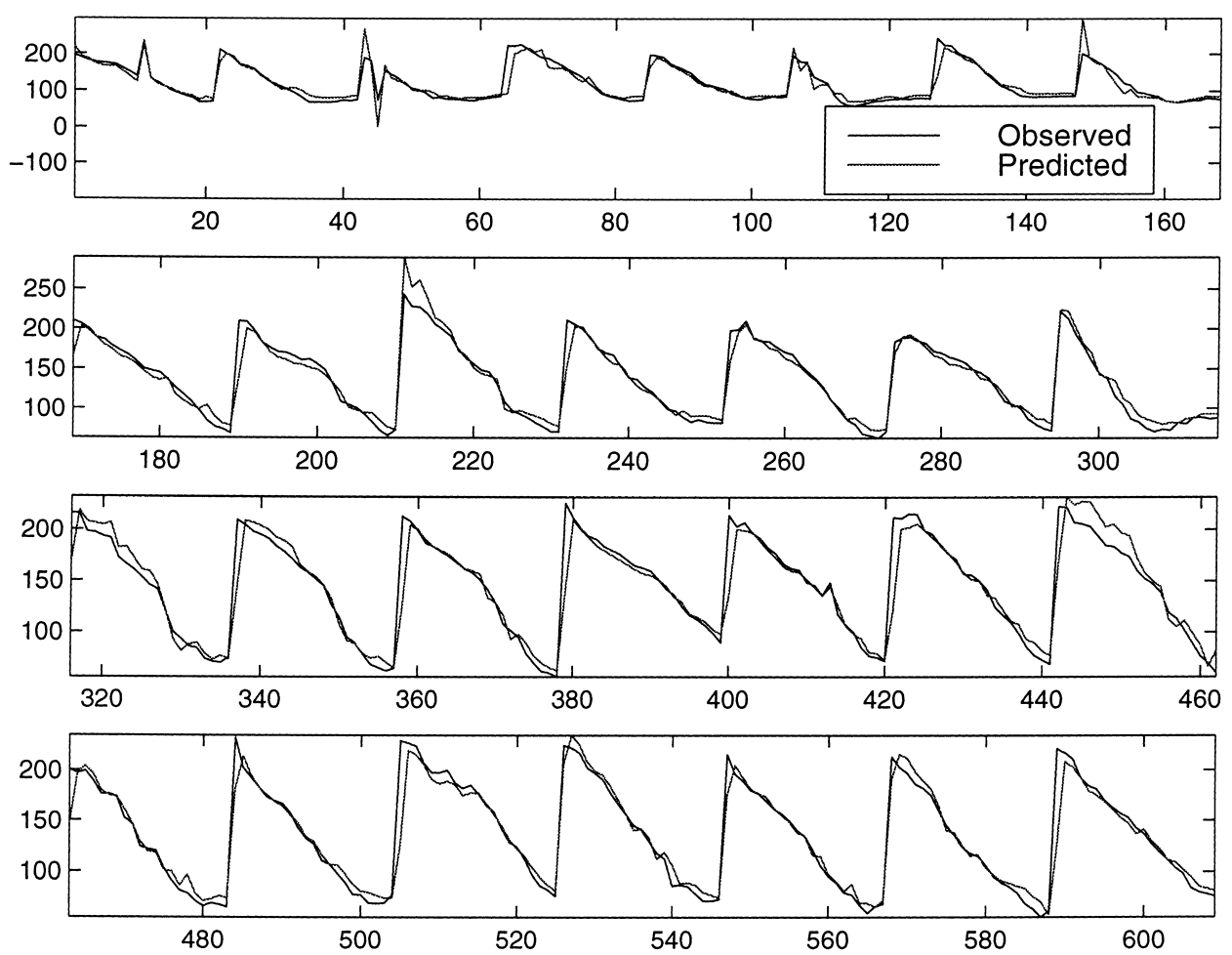

Fig. 1. Dynamic behaviour of glucose after bolus on 29 subjects. The four plots are respectively: healthy-young, healthy-aged, cardiacaged (1-7) and cardiac-aged (8-14).

values of the glucose in the insulin equation are what is to be expected in the human body, where no term can have just an instantaneous effect.

Indeed the fit of the minimal model for each group as an entity is quite good, but not comparable to the new model. On the other hand, if each individual is estimated singly, a great confusion results in the values of the parameters between each group and in the values of the sensitivity index. Furthermore the value of the coefficients $p_{1}, p_{3}$ or $b_{1}, b_{3}$ are very small in the order of $10^{-3}$ and $10^{-2}$ respectively so it is difficult to assign statistical significance to the ratio. Moreover for these models, there are instabilities for each group, which become greater as time increases, since the bolus. This gives rise to spurious results and inaccurate diagnostics [37].

Thus the dynamic modelling approach, adopted in this paper, furnishes a more accurate model of the phenomenon under consideration, whose greater complexity is in line with the basic dynamics of the human body, viz. processes have damped lags. In special cases the minimal model may be taken as an approximate model of the phenomenon, since that the two models are not incompatible, although their precision is quite different. Also, if the given model (24) and (25) is taken as the more accurate model, which it must be, then the asymptotic value of the process cannot yield the insulin sensitivity index, for it will depend on the other parameters as it can be easily seen [37]. The insulin sensitivity index can be taken as a rough measure of the pathologies and perhaps in former times it was better than other tests to provide some indication on the possible pathologies. Now, by using the full model, a better assessment of the diagnosis of a particular pathology can be enacted. The equations above can be used to determine the glucose and insulin time curve after the bolus for the different individuals and so obtain an indication of their pathologies. 

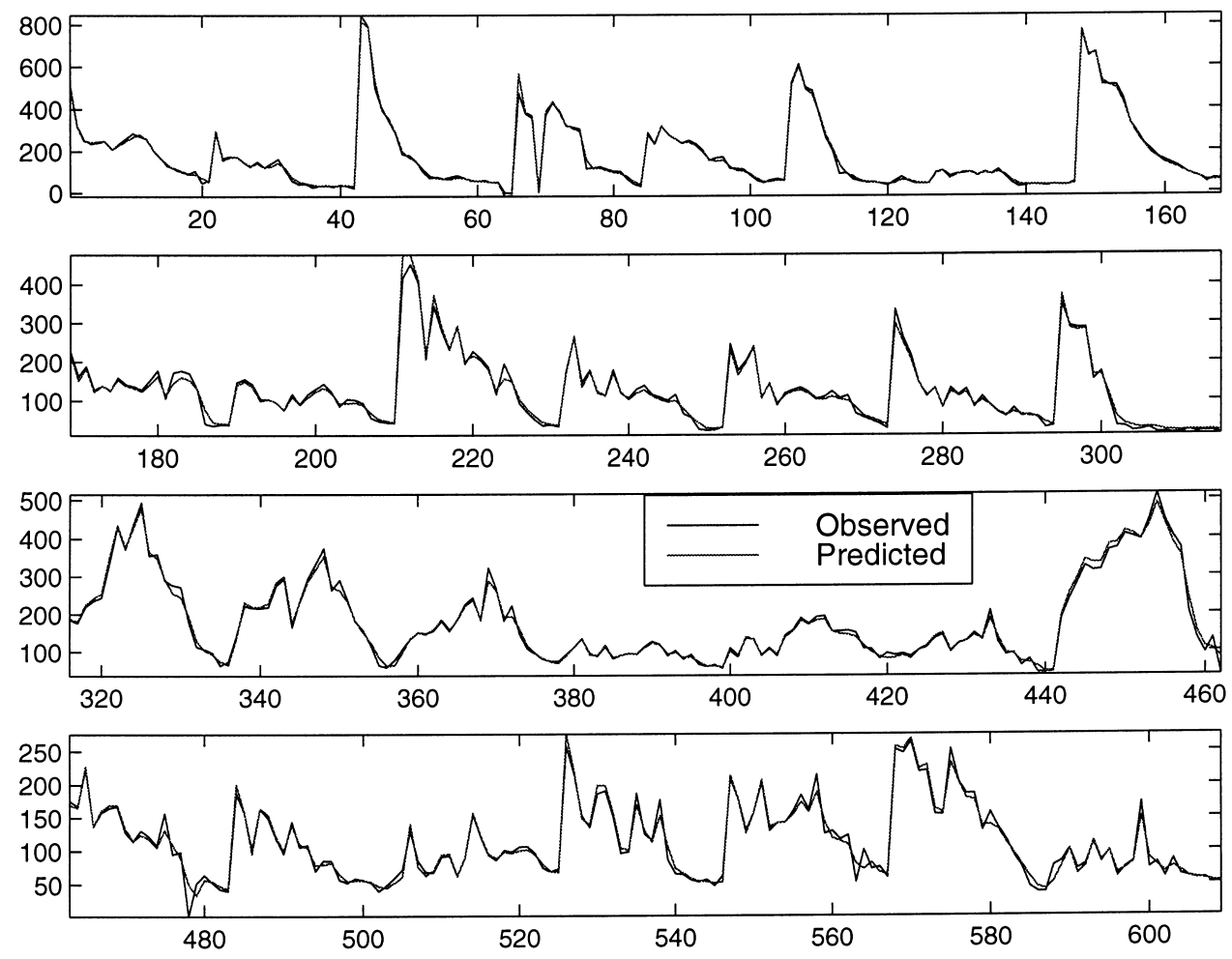

Fig. 2. Dynamic behaviour of insulin after bolus on 29 subjets. The four plots are respectively, healthy-young, healthy-aged, cardiacaged (1-7) and cardiac-aged (8-14).

\subsection{Magnetic resonance imaging segmentation}

Image segmentation is a fundamental problem in image processing and computer vision with numerous applications including, but not limited to, medical image analysis, image compression, etc. Segmented images are caricatures of the original ones which should preserve the most significant features while removing unimportant details. Those images are very easy to display and can be used as a rough representation of the original image. Segmentation is also used as a preprocessing step for image recognition or as an aid in coding images since only the transitions between a few grey levels need to be coded.

The motivation for the research reported in this section lies in the domain of image analysis of volumetric medical images, specifically brain magnetic resonance scans. This methodology has provided a very useful precision tool for information required in fields like reparative surgery, radiotherapy treatment planning, stereotactic neurosurgery, and brain anatomo-functional correlation [52]. The 3D segmentation of white matter, grey matter, Cerebro-Spinal Fluid, bone etc. is extremely important for quantitative analysis such as volume measurements and as a vehicle for gaining new insight into the structural changes of the brain over time and across individuals. Volumetric analysis of cortical and subcortical structures has been found to be useful in assessing progress or remission of various diseases like Alzheimer's disease, epilepsy, etc. Postmortem studies of the human brain reveal consistent age related reductions in brain size and age related increase in Cerebro-Spinal Fluid spaces, which are accentuated in Alzheimer disease [30])

A novel 3D segmentation algorithm which partitions the Magnetic Resonance brain scan into grey matter, white matter, Cerebro-Spinal Fluid, and skin (remaining voxels) based on their grey scale content 
has been presented in $[103,99]$ which can make use of some Operations Research techniques for the segmentation and analysis of three dimensional brain scans. A variety of approaches have been reported for brain image segmentation $[91,52,101,26]$ however, none of them are fully automatic. Segmentation of isodensity regions has been reported in $[26,52]$ but these methods require certain degree of human intervention in achieving the segmentation and therefore are subject to variations due to the experimenter and thus are unreliable for careful experimental studies [103,106]

Statistical methods have become very promising in the recent past since the seminal work of Geman and Geman [40] who modelled images using Markov Random Fields with a superimposed Gibbsian distribution. They adopted a Bayesian approach in image restoration applications and developed the theory and algorithms for the maximum a-posteriori estimation of the original image given the degraded one, using stochastic relaxation and simulated annealing. Moreover they established convergence properties for the algorithm [40]. Since their seminal work, there has been a flurry of activity in applying their technique and variations thereof to various domains including medical image analysis [15,74,101,105,104]. In [15,59,74], Gaussian models have been used for the probability distribution of an observed image given the uncorrupted image of a scene.

The application of a clustering algorithm like the $k$-mean algorithm, to get segmented images, which does not utilise any spatial information contained in an image is not recommendable because this information is very fundamental and must be utilised for effective segmentation. In [75] a clustering algorithm was used to estimate the centre of mass for each class and relaxation was used to get local consistency. The knowledge of the anatomical structure was coupled with certain knowledge about the image formation to get a 3D labelling of a brain Magnetic Resonance Imaging. An attributed graph with nodes corresponding to regions and links corresponding to relations between regions was used to check for consistency from symbolic representation obtained from individual slices. Spatial information may be incorporated in the form of spatial smoothness constraints captured by Markov Random Field models of images. In [15,74], a stochastic model that allows for incorporating spatial smoothness constraints was used with a clustering-based algorithm to achieve image segmentation. The algorithm presented was a generalisation of the $k$-mean clustering algorithm which included spatial constraint in the form of a Markov Random Field and which accounted for local intensity variations of the grey level in the image via local estimate of the region mean grey value. A Hierarchical implementation of the algorithm was also implemented to speed up the convergence of the method. A number of other algorithms has been introduced in the past which applied $k$-mean algorithm and Markov Random Field to image segmentation $[40,15,59]$ but they all required homogeneous values of the grey-level in each region [74]. In [103,106] a $3 \mathrm{D}$ adaption of the $2 \mathrm{D}$ clustering algorithm described in [74] was presented. The $k$-mean clustering algorithm was used to obtain a fully automatic algorithm for 3D image segmentation. The region process was modelled as a Gibbs Random Field (GRF) and a novell definition of the Gibbsian parameter $\beta$ $[103,106]$ analogous to the compatibility function in relaxation labelling algorithms of Hummel et al. [47] was given. The intensity of each region was modelled as a white Gaussian process with mean $\mu_{v}\left(x_{v}\right)$ and variance $\sigma^{2}$, where $\mu_{v}\left(x_{v}\right)$ is the mean grey value of cluster $x_{v}$. Optimisation techniques were then used to get a segmentation $x$ of the volume image.

More specifically, let the observed image at an image location $v$ be denoted by $y_{v}$ and let the actual region label for that location be $x_{v}$. Given that the region process is modelled as a Markov Random Field (MRF), to model the conditional density of the observed image given the distribution of regions, a Gaussian distribution was used, as in [74]. Then, using the Bayes theorem, the a posterior probability density function can be defined as

$$
p(x \mid y) \propto p(y \mid x) p(x),
$$

where $p(x)$ is the Gibbs density and $p(y \mid x)$ is the conditional density of the observed image given the distribution of regions [40]. 
To get an estimate of the distribution of regions, the probability density function $p(x \mid y)$ is maximised. This yields the maximum a-posteriori estimate of $x$. To get a maximum of $p(x \mid y)$ the Gibbs sampler and simulated annealing can be used [40].

In $[74,103,106]$ the maximum was obtained using the iterated conditional mode (ICM) method [15], also known as greedy algorithm. This process converges to a local maximum [15] and the convergence is very fast. The maximisation was performed at every voxel in the image considering the following formula:

$$
\max p\left(x_{v} \mid y_{v} \in V\right) \propto \exp \left\{-\frac{1}{2 \sigma^{2}}\left[y_{v}-m_{v}\left(x_{v}\right)\right]^{2}-\sum_{c \in C} V_{c}(x)\right\},
$$

where the subscript $v$ is index for voxels in a volume image $V$ which is a collection of $M \times N$ slices forming the volumetric structure of the data set, $m_{v}\left(x_{v}\right)$ is an estimate of the mean grey value of the region at voxel $v$, (which is considered as a function of both the voxel $v$ and the cluster $x_{v}$ that the voxel belongs to, therefore its estimation needs to be done locally) $C$ defines the neighbourhood system and $V_{c}(x)$ is the clique potential $[74,40]$.

To apply such a model in a segmentation process, good estimates of $\mu_{v}\left(x_{v}\right)$ and $\sigma_{r}$ are needed. In [74] the problem of $\sigma_{r}$ estimation was not addressed in spite of being acknowledged as a very important parameter in the segmentation process (Pappas assumed that $\sigma_{r}$ was known and equal for each region). In [91] different values of $\sigma$ have been allowed for each region, but the issue of the estimation of $\sigma_{r}$ and $\mu_{r}$ has not been addressed. The fine tuning of the technique they have presented is left to the user who has to tailor the algorithm picking the best choice of $\mu_{r}$ and $\sigma_{r}$ by trial and error.

In [103] the problem of variance estimation has been addressed. A weighted variance has been computed, where each voxel was used for the computation of the variance of a given class and its contribution was weighted by the probability of that voxel belonging to the given class, given the underlying image data (for more details see [103]).

Then the algorithm works in the following way:

1. An initial segmentation of the image is obtained via the $k$-mean algorithm.

2. An estimate of $m_{v}\left(x_{v}\right)$ and $\sigma^{2}$ is obtained (the estimate of $m_{v}\left(x_{v}\right)$ has been obtained via a three-dimensional sliding window of decreasing size, while the estimate of $\sigma^{2}$ has been obtained in a probabilistic framework [103]).

3. Get a new segmentation by

$$
\max _{x} p(x \mid y)
$$

4. If there are changes in the segmentation go to 2, otherwise stop.

This technique has been applied to medical image data yielding visually good segmentation. The quality of the results could be further improved using better imaging techniques such as application of specific pulse sequences which can enhance the separation between cerebrospinal fluid, grey matter and white matter [75].

The two data sets used for the experiments comprised of sagittal sections of the human brain. Each of the two data sets has an in plane resolution of $(256,256)$ and a slice thickness of $1.25 \mathrm{~mm}$. The first data set contained 30 slices and the second contained 100 slices. In Fig. 3 the results of the segmentation algorithm are compare to Pappas' algorithm [74]. For further details on the implementation of the experiments and for the complete sets of results the reader is referred to [103].

A further development of this technique is to perform the estimation of $\mu_{v}\left[x_{v}\right]$ and $\sigma_{r}$ and the maximisation of $p(x \mid y)$ simultaneously using the S.O.C.R.A.t.E.S. algorithm [61]. This approach seems to be very 


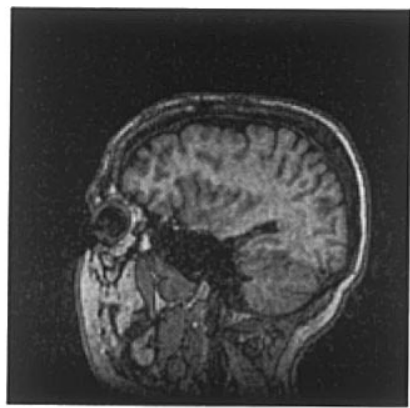

(a)

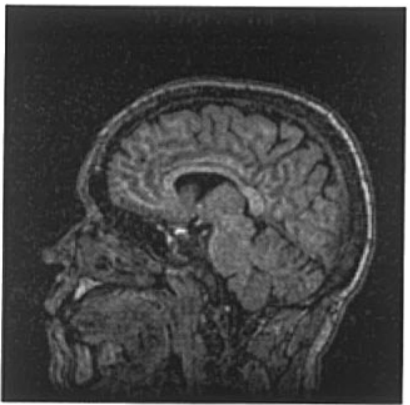

(d)

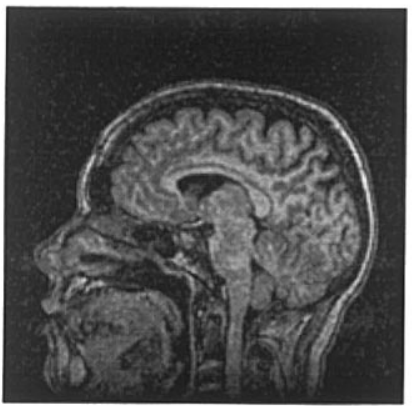

(g)

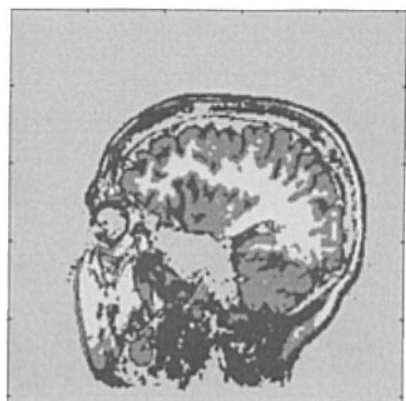

(b)

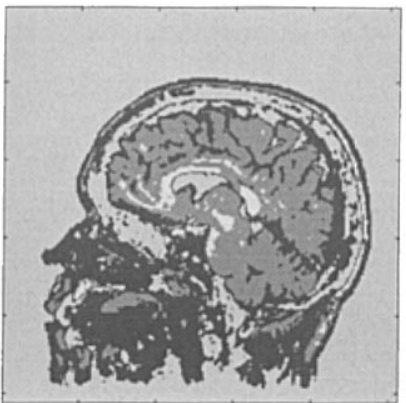

(e)

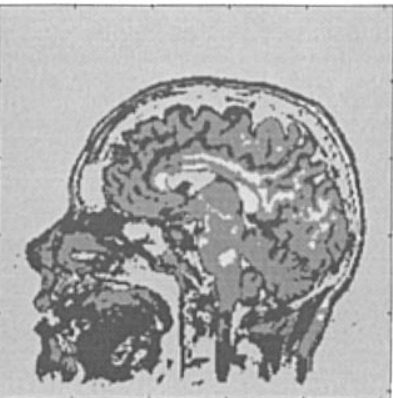

(h)

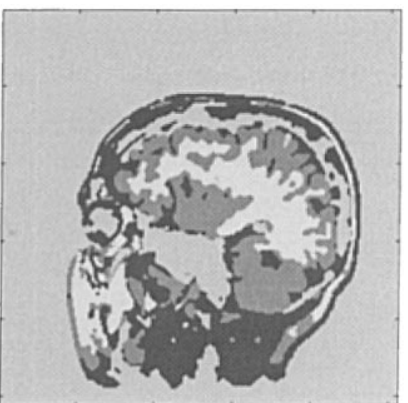

(c)

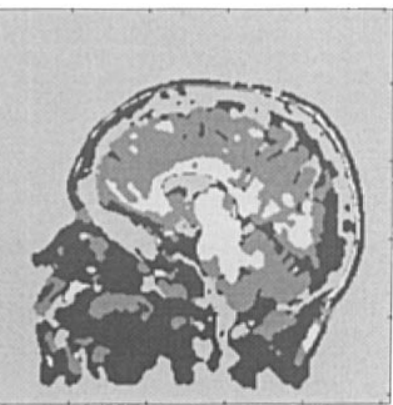

(f)

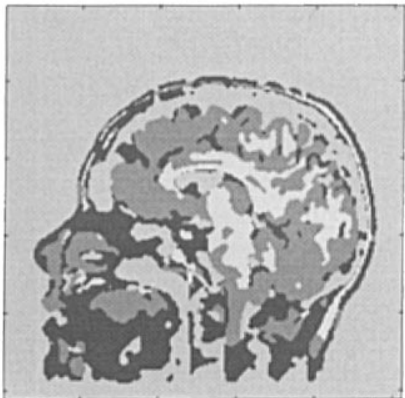

(i)

Fig. 3. Automatic segmentation vs. Pappas' algorithm. (a), (d), (g): Slices of original data from 100-slices dataset. (b), (e), (h): Corresponding slices from 3D segmentation using $\beta$ and iterative variance estimation. (c),(f),(i): Corresponding slices from the $2 \mathrm{D}$ segmented image using Pappas' algorithm with $\beta=0.5$ and $\sigma=5$.

successful [68] and has been applied to a number of problems. In fact, in general, image processing techniques may be formalised as operations research problems by defining a suitable objective function and suitable constraints so that algorithms such as Se.N.E.C.A. [78] and S.O.C.R.A.t.E.S. [60,79] can be applied to estimate and optimise simultaneously. 


\subsection{Analysis of heart dynamics}

A particularly important problem regarding how the heart functions is to understand how the heart beat is controlled and how its frequency and pumping capacity varies throughout the day. An obvious starting point is to try to model the blood pressures and the heart rate as a dynamic system identified from the data. Thus the aim of this section is to describe such a model.

Signals of cardiovascular origin though almost periodical are characterised by slight cycle to cycle variations (oscillations) in both amplitude and time duration. A discrete series describing these oscillations either as functions of cardiac cycles or equivalently, as time functions obtained from these variables by means of interpolation techniques are generally referred to as variability signals.

The wave amplitude variations have different cyclical patterns not only synchronous with breathing activity, but also with longer periods of about 10-20 beat duration, referred to as Mayer waves [82]. These short duration rhythms are normally present and superimposed on to slower ones, consisting in very long period oscillations, e.g. circardian rhythm.

The heart rate variability signal seems capable of contributing to the fundamental investigation of various pathophysiological states: hypertension, diabetes ischemic heart disease. The techniques of analysis have included: Fourier analysis [48,92], linear difference equations [116], spectral analysis techniques $[9,86]$ and various other methods.

This type of analysis is important also for a quantitative evaluation of the role of the autonomic nervous system in the genesis of these rhythms, as it can be seen, for instance, in the analysis of the spectra (power and frequency of the variability components). Particularly the rhythm with a period of nearly 10 seconds seems to be a marker of the interaction between the sympathetic and the parasympathetic systems [57]

For the experiment, the Systolic, Diastolic and Mean blood pressure was obtained from an electrocardiogram and the heart beat duration was determined as the interval between two consecutive $\mathrm{R}$ waves, as they are called. Further the lung filling of the patient was measured with a suitable apparatus. The objective of the model was to determine the future heart beat rates, given these time series, measured on the heart beat intervals, which are just under 1 second, so as to evaluate the role of the autonomous nervous system and verify the periodicity of the Mayer waves. For the patient 2882 beats were recorded and these are given in Fig. 4.

Let the following symbols be defined:

$R_{t}$ - heart beat duration at time $t$,

$S_{t}$ - Systolic blood pressure at time $t$,

$D_{t}$ - Diastolic blood pressure at time $t$,

$M_{t}$ - Mean blood pressure at time $t$,

$L_{t}$ - Lung fill volume at time $t(\mathrm{ml})$

then the dynamic system that was determined with the Se.N.E.C.A. algorithm [78] is given by

$$
\begin{aligned}
R_{t}= & -0.5822+2.5216 S_{t}+4.4686 D_{t}-4.9935 M_{t}+31.7122 L_{t} \\
& +0.706 R_{t-1}+0.1621 S_{t-1}+0.1750 D_{t-1}-0.0636 M_{t-1}-0.0469 L_{t-1} \\
& +2.9098 R_{t-2}-5.3197 D_{t-2}+0.3877 L_{t-2}-1.3290 D_{t-3}-1.0571 M_{t-3} \\
& -0.9302 L_{t-3} 1.9116 R_{t-4}+1.9874 D_{t-4}+11.2252 R_{t-5}-5.7128 S_{t-5} \\
& +1.6189 M_{t-5}-1.7522 L_{t-5}+0.1500 L_{t}\left(R_{t-1}-R_{t-2}\right) \\
& -0.0052 R_{t-1}\left(S_{t-2}-S_{t-3}\right)-0.0017 R_{t-1}\left(D_{t-2}-D_{t-3}\right) \\
& -0.0680 D_{t}\left(S_{t-1}-S_{t-2}\right)+0.00002 S_{t} D_{t} M_{t}-0.0032 S_{t} M_{t} L_{t} .
\end{aligned}
$$

The actual and estimated curve for the heart beat rate is given in Fig. 4. 

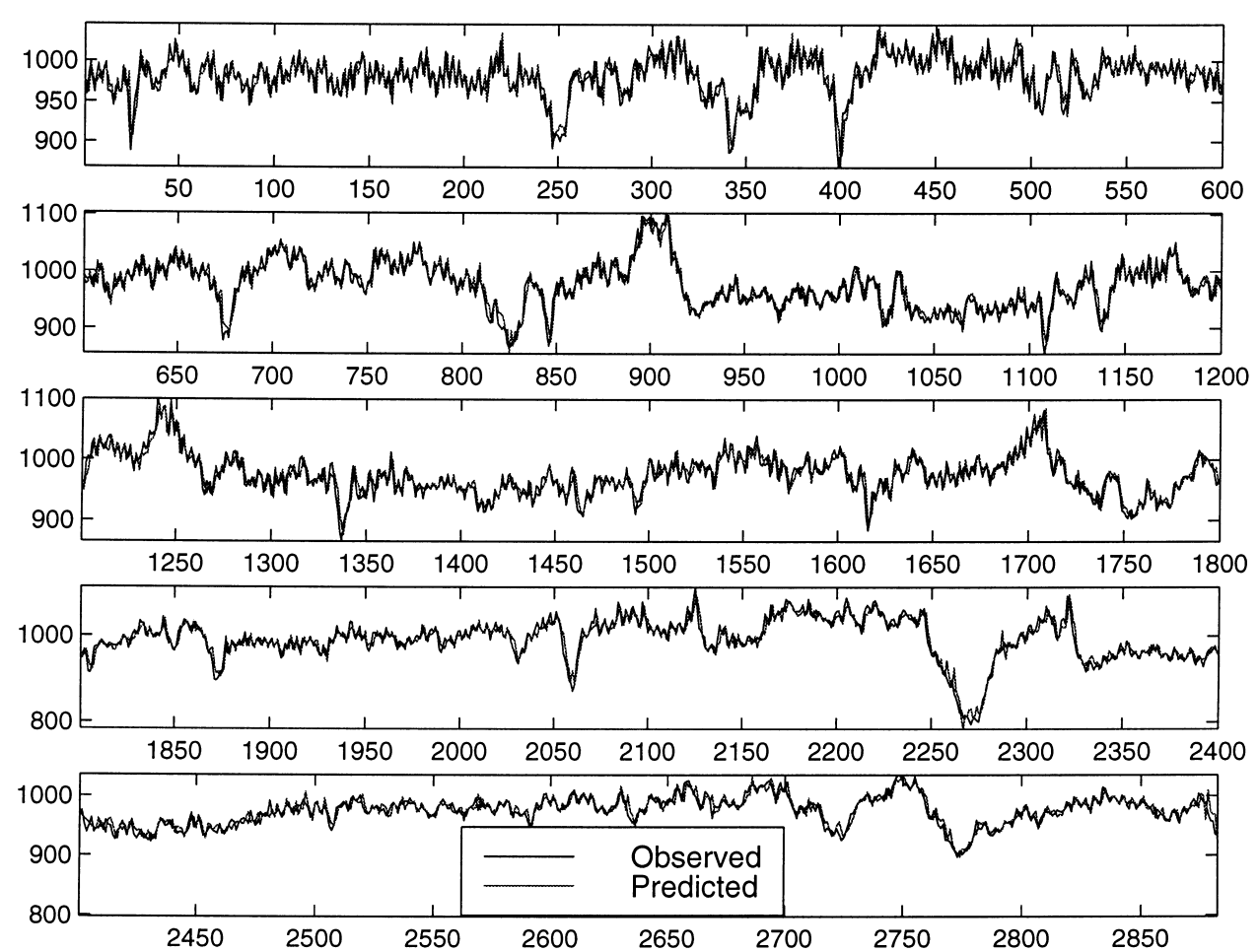

Fig. 4. Actual and predicted heart rate time series (2882 beats), interval in milliseconds.

The fit of the heart rate is very good and the model shows that indeed the heart beat rate forms a highly nonlinear system in the chosen variables. Notice the missing terms and the rather long lags. This could be a measure of the time required to send arterial blood to the perifery, await the message returned by the nervous system and provide a synthesis of the next beat, so as to furnish sufficient blood in the peripherical limbs. However, this is rather conjectural and further research is to be expected on this point. In this model there is no sign of the Mayer waves, as there is no recurrent 10 period lagged items.

The dynamic analysis of this system is important [36] and reveals that the system is stable, which implies that the lagged control is very efficient. However, given the length of the lags and the coefficients found, deviations of the latter, appropriate changes, even small, in the values of the estimated parameters can render the system unstable. It would be therefore extremely interesting to repeat the experiment for cardiac patients and other pathological groups, to determine whether the heart beat rate and the model structure vary significantly from group to group. If so, how is the controllability, reachability, observability or stability of the system altered. In this case a very useful tool would be available, over and above the existing ones, to diagnose heart disease.

\subsection{Optimisation in hyperthermic treatment}

There is experimental evidence on the effectiveness of deep hyperthermia [65], i.e. heating of deep seated tumours above $43^{\circ} \mathrm{C}$. The goal of a treatment is to give an effective thermal dose to the entire tumour. In all tumour cells the temperature should be $43-45^{\circ} \mathrm{C}$ for a period of at least 1 hour per session. At the same time normal tissue temperatures should remain below $41^{\circ} \mathrm{C}$ to prevent thermal damage. The therapeutic effi- 
ciency of a hyperthermic treatment is characterised by the temperature distribution in the tumour above $43^{\circ} \mathrm{C}$ and the lowest temperature in the tumour. Several authors support these conclusions for the clinical outcome of thermoradiotheraphy [72,100], which includes both hyperthermia and radiation treatment. Conversely, tolerance by the patient is limited by the total absorbed power and local discomfort.

Biological research has clearly demonstrated the tumour killing potential of hyperthermia. Two separate mechanisms are responsible for this tumour cell death:

1. hyperthermia enhances the sensitivity of tumour cells to radiation. Maximum enhancement is achieved if both modalities are applied simultaneously;

2. hyperthermia alone is capable of killing tumour cells, especially if the $\mathrm{pH}$ and nutrients in the tumour cells are low, which occurs under fairly common hypoxic conditions in the tumour.

The latter effect is clinically interesting because often tumour cells in such an environment are difficult to reach by chemotherapy and less sensitive to radiation than well oxygenated tumour cells.

Current applications of hyperthermia in the clinic are made possible by the development of adequate heating equipment. The size and location of the tumour has a significant impact on applicator design and on the type of heating. Deep seated tumours require increased heating penetration. For electromagnetic devices this can be achieved by selecting a lower frequency, but then it is no longer possible to focus energy into a small region due to the longer wavelength.

A phased array of antennas positioned around the patient is a widely used method for deep heating. By changing the frequency, the phase and the amplitude of the individual antennas the deposit of energy within the patient can be optimised, while at the same time respecting the temperature constraints on the healthy tissue.

In fact, avoiding hot spots in normal tissue is a major problem with these systems. Due to fringing fields, hot spots occur frequently on the skin near the outer boundary of the water bolus which is placed between the applicator and the patient, but also at deeper locations caused by interference between applicators. Phase steering tends to produce power maxima not just in the target region, but also elsewhere which should be avoided.

Thus the fundamental problem is to determine methods to apply the energy in the target region, so as to heat the tumour as much as possible, while keeping all healthy tissue at physiological temperatures.

The optimisation problem to be solved is therefore concerned with maximising therapeutic efficiency with a given power level, which will depend on the position of the antennas, the amplitude and the phase of the radiation. Thus treatment efficiency and interference problems which may occur will depend on the positions of the antennas and the electromagnetic power issued. Moreover, heat effects are lagged non linear functions of these variables. The temperature attained depends on the blood circulation in the given region and thus the difficulty of achieving an effective treatment depends on the position of the tumour.

Recent works on hyperthermia phased-array optimisation discuss the problem of minimising the power delivered to tissues different from the target, by calculating the Specific Absorption Rate (SAR), i.e. the power deposited per unit mass of tissue. In Ref. [96] an attempt is made to minimise the integrated mean square error between the ideal SAR distribution and the calculated SAR delivered to the patient, assuming that the ideal distribution is unity in the tumour region and zero elsewhere. Another approach [16] consists in defining a specific performance indicator given as the ratio between deposited power in the tumour and the total power delivered both to the tumour and surrounding healthy tissues. In Ref. [113] a summary is presented of some recent criteria and strategies useful to increase power or thermal dose assigned to the tumour.

In the paper [6], optimal values for the phases and amplitudes of the applicators are calculated by minimising the weighted sum of a function of the distances between two complex vectors: the first indicating the required values to maximise the averaged SAR in the tumour and the second indicating the values that minimise this average SAR value in the healthy tissues.

A method capable of planning an optimum treatment for deep regional hyperthermia is proposed [97]. Two techniques are presented which utilise the finite difference and time domain (FDTD) method to create 
a data base of information with which the operator can simulate a patient treatment interactively. A way to quantify the output is the ratio between average SAR in the tumour and average SAR in the body.

Other studies on phased arrays consider a different optimisation problem where the objective function is related to the temperature distribution achieved in the patient. Heat balance in perfused tissue is modelled with the Bio-Heat Transfer Equation (BHT) [89], inserting the predicted power deposited as source term. The mean square error between the predicted temperature $T$ and therapeutic temperatures, $43-45^{\circ} \mathrm{C}$ can be minimised, using a penalty function method [70]. Treatment optimisation can also be defined as an unconstrained minimax problem, the objective function being a very realistic one since it considers the minimum tumour temperature [108].

Adaptive finite elements are used [10] in a simulation algorithm, which involves two steps: calculation of electromagnetic fields and the resulting temperature distribution. In both cases a tetrahedral mesh is used for the discretisation of the solution area to get a good resolution of complicated tissue boundaries. For applications, the temperature distributions in the stationary case, resulting from all independent combination of antenna phases and amplitudes have to be computed.

All these methods, except perhaps the last, are essentially static algorithms to determine the appropriate power levels to apply in any given circumstances, which must then be integrated and adapted periodically, by determining the actual distribution of temperatures through a number of probes. The last procedure provides a look-up table of how to alter the power applied depending on the temperature gradients which emerge, the position of the tumour and the position of the antennas. Thus it is really the clinician who will control the heating process, by watching the temperature readings and modifying, as his experience indicates, the frequency, phase and amplitude of the antennas.

However, the heating process can clearly be defined as a dynamical system with control or input variables and a set of constraints to be satisfied through time. By modelling sufficiently accurately the dynamic heating system in terms of the situational environment, the distribution of the temperature throughout the region can be calculated and these can be checked against the actual temperature readings. Moreover by changing the control variables, frequency, phase and amplitude of the antennas an adaptive dynamic system is defined, which will maximise the heat delivered to the tumour while respecting all the relevant constraints imposed. In this case, the clinician will be able to monitor the optimal adaptive control of the system and intervene, if need be, in an appropriately optimal way, based on the optimal response that the system will indicate to an imposed direction of change in the local temperature gradients.

This has been proposed [5] where a non linear dynamic system is optimised through its transformation as a nonlinear optimisation problem with constraints. Control variables of hyperthermic treatment can be determined by maximising temperature in the tumour, subject to constraints on the temperature distribution in the healthy tissues. The algorithm applied to solve the constrained optimisation problem, is for non linear non convex problems [77,81]. This algorithm determines directly Kuhn-Tucker points by solving at each iteration a linear complementarity problem.

The temperature distribution at time $\bar{t}$ is a function of the position of the point, say $r$, being considered, of the phases $\phi$ and the amplitudes $A$. So the following optimisation problem at a certain point of time $\bar{t}$ must be solved:

$$
\begin{aligned}
& \max _{\phi, A} \frac{1}{\operatorname{mis}\left(\Omega^{+}\right)} \int_{\Omega^{+}} T(r, \phi, A) \mathrm{d} r \\
& \text { s.t. } \\
& T(r, \phi, A) \leqslant 41, \quad r \in \Omega^{-}, \\
& T(r, \phi, A) \geqslant 43, \quad r \in \Omega^{+}, \\
& -180^{\circ} \leqslant \phi \leqslant 180^{\circ}, \\
& \sum_{k} A_{k}^{2}=1
\end{aligned}
$$


where $\Omega^{+}$denotes tumour region, $\Omega^{-}$denotes the region of healthy tissue. The number of the cells in the lattice which are contained in subdomain $\Omega^{+}$, is indicated by $\operatorname{mis}\left(\Omega^{+}\right)$.

The space discretisation described [81] produces the following two-dimensional optimisation problem:

$$
\max _{\phi, A} \sum_{(i, j) \in \Omega^{+}}\left(\operatorname{cost}(i, j)+\sum_{h, k} c_{h, k}(i, j) A_{h} A_{k} \cos \left(\phi_{h}-\phi_{k}\right)+\sum_{h, k} \mathrm{~d}_{h k}(i, j) A_{h} A_{k} \sin \left(\phi_{h}-\phi_{k}\right)\right)
$$

subject to:

$$
\begin{aligned}
& \operatorname{cost}(i, j)+\sum_{h, k} c_{h, k}(i, j) A_{h} A_{k} \cos \left(\phi_{h}-\phi_{k}\right)+\sum_{h, k} \mathrm{~d}_{h k}(i, j) A_{h} A_{k} \sin \left(\phi_{h}-\phi_{k}\right) \leqslant 41, \quad(i, j) \in \Omega^{-}, \\
& \operatorname{cost}(i, j)+\sum_{h, k} c_{h, k}(i, j) A_{h} A_{k} \cos \left(\phi_{h}-\phi_{k}\right)+\sum_{h, k} \mathrm{~d}_{h k}(i, j) A_{h} A_{k} \sin \left(\phi_{h}-\phi_{k}\right) \geqslant 43, \quad(i, j) \in \Omega^{+}, \\
& -180^{\circ} \leqslant \phi_{h} \geqslant 180^{\circ}, \quad h=1, \ldots, N, \\
& \sum_{k} A_{k}^{2}=1 .
\end{aligned}
$$

Subscripts $h$ and $k$ represent the electromagnetic applicators, cost $(i, j), c_{h k}$ and $d_{h k}$ are parameters determined by numerical solving of Bioheat Equation, which models thermal exchanges in biological tissues [77].

Optimisation algorithm G.O.Al. [77,81] beginning with an arbitrary initial value produces a set of feasible solutions until it finds local optimal solutions. By finding successive approximate Kuhn-Tucker points, through the linear complementarity problem routine, and by shifting appropriately the trust region constraints, decreasing local stationary points are determined, until a Kuhn-Tucker point is found, whose solution yields the global minimum. Thus the procedure can be considered as finding successively lower Kuhn-Tucker points by bounding the ones already found.

To give an idea of the complexity of the problem we reproduce specimen solution from one run of G.O.Al. [77,81], where many local maxima were found. In Table 1, three local maxima with the associated temperatures in the tumour and in the healthy tissues exposed to heating are shown. Also the relevant control variables are indicated.

Table 1

Local maxima

\begin{tabular}{llll}
\hline & First local maximum & 10th local maximum & Best local maximum \\
\hline Temperature in the tumour & 39.17 & 43.9 & 45.65 \\
Temperature in healthy tissue & 37.3 & 38.1 & 38.3 \\
Control Variables & & & 0.744 \\
Amplitude Applicator 1 & 0.373 & 0.699 & 0.194 \\
Amplitude Applicator 2 & 0.097 & 0.175 & 0.157 \\
Amplitude Applicator 3 & 0.089 & 0.148 & 0.694 \\
Amplitude Applicator 4 & 0.349 & 0.624 & 6.6 \\
Phase Applicator 1 & 6.99 & 6.768 & 8.3 \\
Phase Applicator 2 & 8.43 & 8.37 & 31.0 \\
Phase Applicator 3 & 29.75 & 31.30 & 0.0 \\
Phase Applicator 4 & 0.0 & 0.0 & \\
\hline
\end{tabular}


Application of the algorithm G.O.Al to the problem [5] has given as an optimal local maximum the following result: $A=(0.744,0.194,0.157,0.694), \phi=(6.6,8.3,31.0,0.0)$. These control variables correspond to average temperature in the tumour of $45.6^{\circ} \mathrm{C}$, this temperature is in the therapeutic range. The average temperature in the healthy tissue is $38.3^{\circ} \mathrm{C}$, so we have an appreciable protection.

This research [8] is now undergoing generalisation to $3 \mathrm{D}$ and the parameters, the optimal heating and the exact location of the probes are determined by and optimisation algorithm at the same time, S.O.C.R.A.t.E.S. [79]. Period by period these quantities are adaptively improved, until the calculated and actual values differ less then the precision criterion imposed [79]. Of course exogenous controls can be enacted and the system will respond optimally.

\section{Conclusions}

The modelling of natural phenomena by nonlinear dynamic systems appear to be a natural way of presenting such phenomena and the modern techniques that can be applied seem very promising.

Certainly this approach seems to give rise to highly nonlinear systems with rather long lags, which appears, from the little we know, in line with natural systems. The algorithms described in this review seem however to be capable of handling these complex problems and from the examples given, it can be seen that they do a good job in the modellisation and in their control.

Obviously, much research needs to be done in these dynamic aspects of modelling. Only the future will tell how far these systems can be considered a success. However, the aspects discussed here, it seems important to stress:

- The modelling of the phenomena should not be adapted or simplified to fit the computational techniques available, since this will give rise to incorrect, biased and limited models.

- Rather the specification and the identification of the system should be data driven, rather than theory driven. If the data fits the theory, it is all to the good, if it does not, it is important to determine in what aspects they differ.

- The dynamic structure is crucial to determine both the fit of the data to the model and the properties that it should exhibit. Again discrepancies in the dynamic structure implies that the model is incorrect.

In conclusion, this review has been designed to present some efforts that have been done to try to represent phenomena by suitable dynamic models. This is a very important field of research, as the examples here indicated show, but the research involved is still very much an open question.

\section{References}

[1] T. Amemiya, Advanced Econometrics. Basil Blackwell, Oxford, 1985.

[2] E. Angel, R.E. Bellman, Dynamic Programming and Partial Differential Equations. Academic Press, New York, 1972.

[3] M. Aoki, Optimal Control and System Theory in Dynamic Economic Analysis. North-Holland, New York, 1976.

[4] S.P. Banks, Mathematical Theories of Nonlinear Systems. Prentice-Hall, New York, 1985.

[5] F. Bardati, F. Bartolozzi, G. Patrizi, A constrained optimization approach to the control of a phased array radiofrequency hyperthermia system. Ricerca Operativa 28 (1998) 5-36.

[6] F. Bardati, A. Borrani, A. Gerardino, G.A. Lovisolo, SAR optimization in a phased array radiofrequency hyperthermia system, IEEE Transactions on Biomedical Engineering 42 (1995) 1201-1207.

[7] M.S. Bartlett, On the theoretical specification and sampling properties of autocorrelated time-series, Journal of the Royal Statistical Society (Suppl.) 7 (1946) 211.

[8] F. Bartolozzi, Mathematical programming techniques applied to the adaptive control of phased array radiofrequency hyperthermia system. PhD dissertation. University of Rome, La Sapienza, Dipartimento di Statistica Statistiche Applicate e Probabilità, March 1999. 
[9] G. Baselli, S. Cerutti, S. Civardi, D. Liberati, F. Lombardi, A. Malliani, M. Pagani, Spectral and cross-spectral analysis of heart rate and arterial blood pressure variability signals, Computers and Biomedical Research 19 (1986) 520-534.

[10] R. Beck, H.C. Hege, M. Seebass, P. Wust, P. Deuflhard, R. Felix, Adaptive finite element codes for numerical calculations in hyperthermia treatment planning. In: Atti del VII ICHO, Roma pp. 9-13 (1996).

[11] J. Beishorn, G. Peters (Eds.), Systems Behaviour. Open University Press, London, 1972.

[12] R.E. Bellman, Dynamic Programming. Princeton University Press, Princeton, NJ. 1957.

[13] E.N. Bergman, S.S. Reulwein, R.E. Corlett, Effects of obesity on insulin sensitivity and responsiveness in sheep, American Journal of Physiology 257 (1989) 772-781.

[14] R.N. Bergman, Y.Z. Ider, C.R. Bowdenand, C. Cobelli, Quantitative estimation of insulin sensitivity, American Journal of Physiology 236 (1979) 667-677.

[15] J. Besag, On statistical analysis of dirty pictures, Journal of the Royal Statistical Society B 48 (3) (1986) $259-302$.

[16] A. Boag, Y. Leviatan, A. Boag, Analysis and optimization of waveguide multiapplicator hyperthermia systems, IEEE Transactions on Biomedical Engineering 40 (1993) 946-952.

[17] V.G. Boltyanskii, Optimal Control of Discrete System. Wiley, New York, 1978.

[18] T.S. Brausch, A.R. Pagan, A simple test for heteroschedasticity and random coefficient variation, Econometrica 47 (1979) $1287-$ 1294.

[19] T.A. Buchanan, G.F. Sipos, S. Gadalahand, K.P. Yip, D.J. Marsh, W. Hsueh, R.N. Bergman, Glucose tolerance and insulin action in rats with renovascular hypertension, Hypertension 18 (1991) 341-347.

[20] H. Bunke, O. Bunke, Statistical Inference in Linear Models. Wiley, New York, 1986.

[21] R.P. Buyalos, M.E. Geffner, N. Berschand, H.L. Judd, R.M. Watanabe, R.N. Bergman, Insulin and insulin-like growth factorirresponsiveness in polycystic ovarian syndrome, Fertility and Sterility 57 (1992) 796-803.

[22] M.D. Cannon, C.D. Cullum, Jr., E. Polak, Theory of Optimal Control and Mathematical Programming. McGraw-Hill, New York, 1970.

[23] J.L. Casti, Dynamical Systems and their Applications. Academic Press, New York, 1977.

[24] J.L. Casti, Nonlinear System Theory, Academic Press, New York, 1985.

[25] M. Chen, R.N. Bergman, P.G. Pacini, D. Porte Jr., Pathogenesis of age-related glucose intolerance in man. Insulin resistance and decreased beta-cell function, Journal of Clinical Endocrinology Metabolism 60 (1985) 13-20.

[26] H.E. Cline, C.L. Doumulin, H.R. Hartand, W.E. Lorensen, S. Ludke, 3-D reconstruction of the brain from magnetic resonance images using a connectivity algorithm, Magnetic Resonance Imaging 5 (1987) 345-352.

[27] E.A. Coddington, N. Levinson, Theory of Ordinary Differential Equations, McGraw-Hill, New York, 1955.

[28] R. Courant, D. Hilbert, Methods of Mathematical Physics, Wiley, New York, 1953.

[29] W.S. Cutfield, R.N. Bergman, R.K. Menon, M.A. Sperling, The modified minimal model: Application to measurement of insulin sensitivity in children, Journal of Clinical Endocrinology Metabolism 70 (1990) 1644-1650.

[30] C. DeCarli, J. Moisog, D.G.M. Murphy, D. Teichberg, S.I. Rapoport, B. Horwitz, Method of quantification of brain ventricular and subarachnoid CSF volumes for MRI images, Journal of Computer Assisted Tomography 16 (2) (1992) $274-284$.

[31] R.A. Defronzo, J.D. Tobin, R. Andres, Glucose clamp technique: A method of quantifying insulin secretion and resistance, American Journal of Physiology 237 (1979) 214-223.

[32] S.E. Dreyfus, Dynamic Programming and the Calculus of Variations. Academic Press, New York, 1965.

[33] P. Dyer, S.R. McReynolds, The Computation and Theory of Optimal Control. Academic Press, New York, 1979.

[34] T.L. Friesz, D. Bernstein, T.E. Smith, R.L. Tobin, B.-W. Wie, A variational inequality formulation of the dynamic network user equilibrium problem, Operations Research 41 (1993) 179-191.

[35] A. De Gaetano, Mathematical modelling of the glucose-insulin system. Projet de Thèse de Doctorat mention mathématiques appliquées, Université de Pau et es Pays de l'Adour, December 1995.

[36] A. De Gaetano, E. Di Lena, A. Mondello, S. Marino, G. Patrizi, Nonlinear Dynamical Modelling of Certain Pathological States in Humans. submitted for publication, 1997.

[37] A. De Gaetano, S. Marino, G. Patrizi. Data Driven Modelling by Se.N.E.C.A. (Sequential Nonlinear Estimation by a Constrained Algorithm): The glucose insulin dynamic system. to appear, 1999.

[38] A.R. Gallant, H. White, A Unified Theory of Estimation and Inference for Nonlinear Statistical Models. Basil Blackwell, Oxford, 1988.

[39] R.V. Gamkrelidze, Principles of Optimal Control Theory. Plenum Press, New York, 1978.

[40] S. Geman, D. Geman, Stochastic relaxation, Gibbs distributions, and the Bayesian restoration of images, IEEE Transactions on Pattern Analysis and Machine Intelligence 6 (6) (1984) 721-741.

[41] W.A. Greuver, E. Sachs, Algorithmic Methods in Optimal Control, Pitman, London, 1981.

[42] S.M. Haffner, M.P. Stern, J. Dunn, M. Mobley, J. Blackwell, R.N. Bergman, Diminished insulin sensitivity and increased insulin response in non-obese non-diabetic Mexican Americans, Metabolism 39 (1990) 842-847.

[43] S.M. Haffner, M.P. Stern, R.M. Watanabe, R.N. Bergman, Relationship of insulin clearance and secretion to insulin sensitivity in non-diabetic Mexican Americans, European Journal of Clinical Investigation 22 (1992) 147-153. 
[44] L. Hasdorf, Gradient Optimization and Nonlinear Control. Wiley, New York, 1976.

[45] Sir John Hicks, A Contribution to the Theory of the Trade Cycle. Clarendon Press, Oxford, 1950.

[46] J.A. Howard, J.N. Sheth, The Theory of the Buyer Behavior. Wiley, New York, 1969.

[47] R.A. Hummel, S.W. Zucker, On the foundation of relaxation labelling process, IEEE Transactions on Pattern Analysis and Machine Intelligence 5 (3) (1983) 267-287.

[48] B.W. Hyndman, R.I. Kitney, B. McA Sayers, Spontaneous rythms in physiological control systems, Nature 233 (1974) 339-341.

[49] R.I. Jennrich, Asymptotic properties of non-linear least squares estimators, Annals of Mathematics and Statistics 40 (1969) 633643.

[50] R.S. Johannes, Using the ADAP learning algorithm to forecast the onset of diabetes mellitus. In: Proceedings of the Symposium on Computer Applications and Medical Care, IEEE Computer Society Press, pp. 261-265, 1988.

[51] C. Johnston, P. Raghu, D.K. McCulloch, J.C. Beard, W.K. Ward, L.J. Klaff, B. McKnight, R.N. Bergman, J.P. Palmer, Betacell function and insulin sensitivity in nondiabetic HLA-identical siblings of insulin-dependent diabetics, Diabetes 36 (1987) 829837.

[52] M. Joliot, B.M. Majoyer, Three-dimensional segmentation and interpolation of magnetic resonance brain images, IEEE Transactions on Medical Imaging 12 (2) (1993) 269-277.

[53] R.E. Kalman, Lectures on Algebraic Systems Theory, Springer, Berlin, 1969.

[54] R.E. Kalman, P.L. Falb, M.A. Arbib, Topics in Mathematical System Theory, McGraw-Hill, New York, 1969.

[55] A. Kautzy-Willer, G. Pacini, B. Niederle, G. Schernthaner, R. Prager, Insulin secretion insulin sensitivity and hepatic insulin extraction in primary hyperthyroidism before and after surgery, Clinical Endocrinology Oxford 37 (1992) 147-155.

[56] Sir Maurice Kendall, Multivariate Statistics. Griffin, London, 1975.

[57] R.I. Kitney, O. Rompelman, The Study of Heart Rate Variability. Clarendon Press, Oxford, 1980.

[58] Y.T. Kruszynska, D.S. Harry, R.N. Bergman, N. McIntyre, Insulin sensitivity insulin secretion and glucose effectiveness in diabetic and non-diabetic cirrhotic patients, Diabetologia 36 (1993) 121-128.

[59] S. Lakshmanan, H. Derin, Simultaneous parameter estimation and segmentation of Gibbs random fields using simulated annealing, IEEE Transactions on Pattern Analysis and Machine Intelligence 11 (1989) 799-813.

[60] K. Lazzarini, P. Patriarca, G. Patrizi, Applicazioni di sistemi di identificazione ed ottimizzazione simultanei alla gestione di sistemi dinamici. in: Proceedings of Sugitalia: XI Convegno Utenti Italiani di SAS System, Firenxe, pp. 468-475, Milano, October 25-27th 1995. SAS Institute Srl.

[61] K. Lazzarini, G. Patrizi, Identificazione ed ottimizzazione simultanea di Sistemi dinamici. in: Proceedings of Sugitalia: XI Convegno Utenti Italiani di SAS System, Firenxe, pp. 311-324, Milano, October 25-27th 1995, SAS Institute Srl.

[62] B. Ludvik, M. Clodi, A. Kautzky-Willer, M. Capek, E. Hartter, G. Pacini, R. Prager, Effect of daxamethasone on insulin sensitivity islet amyloid polypeptide and insulin secretion in humans, Diabetologia 36 (1993) 84-87.

[63] E. Mailnvaud, Méthodes Statistiques de l'Économétrie. Dunod, Paris, 3eme (Ed.), 1978.

[64] G. Marchesini, G. Pacini, G. Bianchi, D. Patrono, C. Cobelli, Glucose disposal beta-cell secretion and hepatic insulin extraction in cirrhosis: A minimal model assessment, Gastroenterology 99 (1990) 1715-1722.

[65] C. Marino, B. Avella, L. Galloni, Deep Localized Hyperthermia: Effects on Murine Normal-Tissue. in: Abstracts of 13th ESHO Conference, p. 33ff, Bruxelles, June 1993.

[66] J.G. Miller, Living Systems. McGraw-Hill, New York, 1978.

[67] R.R. Mohler, Nonlinear Systems. Prentice-Hall, New York, 2 volumes, (1991).

[68] L. Nieddu, Image processing via optimization algorithms. PhD dissertation, University of Rome, Dipartmento di Statistica, Probabilitá Statistiche Applicate, 1999.

[69] L. Nieddu, G. Patrizi, Formal properties of pattern recognition algorithms: A review. European Journal of Operational Research 1999.

[70] K.S. Nikita, N. Maratos, N.K. Uzunoglu, Optimum excitation of phases and amplitudes in a phased array hyperthermia system, International Journal of Hyperthermia 8 (1992) 515-528.

[71] R. Nosadini, S. Del Prato, A. Tiengo, E. Dune, G. Toffolo, C. Cobelli, P.P. Foranato, P. Moghetti, M. Muggeo, Insulin sensitivity binding and kinetics in pancreatogenic and type I diabetes, Diabetes 31 (1980) 346-355.

[72] J.R. Oleson, M.W. De Whirst, J.M. Harrelson, K.A. Leopold, T.V. Samulski, C.Y. Tso, Tumour temperature distributions predict hyperthermia effects, International Journal of Radiation Oncology, Biology, Physics 16 (1989) 559-570.

[73] G. Pacini, A. Valerio, F. Beccaro, R. Nosadini, C. Cobelli, G. Crepaldi, Insulin sensitivity and beta-cell responsivity in elderly subjects with normal OGTT, Journal of the American Geriatric Society 36 (1988) 317-323.

[74] T.N. Pappas, An adaptive clustering algorithm for image segmentation, IEEE Transactions on Signal Processing 40 (4) (1992) 901-914.

[75] B. Parvin, W. Johnston, D. Roselli, Pinta: A system for visualizing the anatomical structures of the brain from MR imaging. in: Proceedings of the IEEE Computer Society Conference on computer vision and pattern recognition, pp. 615-616, IEEE Computer Society Press, 15-17 (1993). 
[76] G. Patrizi, Nonlinear dynamics and the long-range planning process of a firm. in: M. Galeotti, M. Geronazzo, L. Gori, (Eds.), Nonlinear Dynamics in Economics and Social Sciences, pp. 231-258, Pitagora Editrice, Bologna, 1988.

[77] G. Patrizi, A New Algorithm for General Nonlinear Constrained Optimization: Convergence and Experimental Results, 1998 (to be published).

[78] G. Patrizi, Se.N.E.C.A. Sequential Nonlinear Estimationby a Constrained Algorithm, 1999 (to appear).

[79] G. Patrizi, S.O.C.R.A.t.E.S. Simultaneous Optimal Control by Recursive and Adaptive Estimation System, 1999 (to appear).

[80] G. Patrizi, C. Spera, Vector and parallel processing applications of nonlinear optimization algorithms: Design problems and experimental results. in: D.J. Evans, C. Sutti, (Eds.), Parallel Computing: Methods, Algorithms, Applications, pp. 225-236, Adam Hilger, Bristol, 1988.

[81] G. Patrizi, C. Spera, G.O.AL.: A general optimization algorithm. Atti del Tredicesimo convegno AMASES, pp. 677-701, Verona, Settembre 1989.

[82] J. Penaz, Mayer waves: History and methodology, Automedica 2 (1978) 135-141.

[83] P. Cavallo Perin, A. Bruno, C. Cobelli, G. Pacini, G. Niort, L. Boine, M. Goria, G. Pagano, Insulin sensitivity in subacute experimental T3 hyperthiroidism in man, Hormone Metabolism Research 20 (1988) 186-187.

[84] Iu.P. Petrov, Variational Methods in Optimum Control Theory. Academic Press, New York, 1968.

[85] M.G. Piccardo, G. Pacini, M. Rosa, R. Vichi, Insulin resistance in myotonic dystrophy, Enzyme 45 (1991) 14-22.

[86] B. Pomeranz et al, Assessment of autonomic function in humans by heart rate spectral analysis, American Journal of Physiology 248 (1985) 151-153.

[87] L.S. Pontryagin, V.G. Boltyanskii, R.V. Gamkrelidze, E.F. Mishchenko, The Mathematical Theory of Optimal Processes. Wiley, New York, 1962.

[88] P. Raghu, C. Johnston, J.C. Beard, R.N. Bergman, D.K. McCulloch, J.P. Palmer, Reduced insulin sensitivity in nondiabetic HLA-identical siblings of insulin-dependent diabetic subjects, Diabetes 34 (1985) 991-994.

[89] R.B. Roemer, Heat transfer in hyperthermia treatments: Basic principles and application. in: AAPM Conf. Physical Aspects of Hyperthermia, Duke University, 1987.

[90] P.A. Samuelson, The Foundations of Economic Analysis. Harvard University Press, Cambridge, MA, 1947.

[91] G. Sapiro, P.C. Teo, B.A. Wandell, Anatomically consistent segmentation of the human cortex for functional MRI visualization. Technical Report HPL-97-03, Hewlett-Packard Labs., 1997.

[92] B. McA Sayer, Analysis of heart rate variability, Ergonomics 16 (1973) 85-97.

[93] G.A.F. Seber, C.J. Wild, Nonlinear Regression. Wiley, New York, 1989.

[94] T. Söderström, P. Stoica, System Identification, Prentice-Hall, New York, 1989.

[95] A.A.R. Starke, Determination of insulin sensitivity: Methodological considerations, Journal of Cardiovascular Pharmacology 20 (1992) 17-20.

[96] J.W. Strohbehn, E.H. Curtis, K.D. Paulsen, X. Yuan, D.R. Lynch, Optimization of the absorbed power distribution for an annular phased array hyperthermia system, International Journal of Radiation Oncology, Biology, Physics 16 (1989) 589-599.

[97] D. Sullivan, Mathematical model for treatment planning in deep regional hyperthermia, IEEE Transactions on Microwave Theory and Technology 39 (1991) 864-872.

[98] B.A. Swinburn, V.L. Boyce, R.N. Bergman, B.V. Howard, C. Bogardus, Deterioration in carbohydrate metabolism and lipoprotein changes induced by modern high fat diet in Pima Indians and Caucasians, Journal of Clinical Endocrinology Metabolism 73 (1991) 156-165.

[99] G. Toffolo, R.N. Bergman, D.T. Finegood, C.R. Bowden, C. Cobelli, Quantitative estimation of beta cell sensitivity to glucose in the intact organism: A minimal model of insulin kinetics in the dog, Diabetes 29 (1980) 979-990.

[100] R. Valdagni, F.F. Liu, D.S. Kapp, Important prognostic factors influencing outcome of combined radiation and hyperthermia, International Journal of Radiation Oncology, Biology, Physics 15 (1988) 959-972.

[101] D. Vandermeulen, R. Verbeeck, L. Berben, P. Suetens, C. Marchal, Continuous voxel classification by stochastic relaxation: Theory and application to MR imaging and MR angiography. In: Lecture Notes in Computer Science 687: Information Processing in Medical Imaging 13th International Conference IPMI, pp. 487-506, Arizona, 1993.

[102] V.N. Vapnik, The Nature of Statistical Learning Theory. Springer, Berlin, 1995.

[103] B.C. Vemuri, L. Nieddu, Probabilistic algorithms for fully automatic image segmentation: Applications to brain MRI. Technical Report Number TR-97-013, Computer and Information Sciences Department, University of Florida, 1997.

[104] B.C. Vemuri, A. Radisavljevic, Multiresolution stochastic shape models with fractal priors, ACM Transactions on Graphics 13 (2) (1994) 177-207.

[105] B.C. Vemuri, A. Radisavljevic, C.M. Leonard, Multiresolution stochastic 3D shape models for image segmentation. In 13th International Conference IPMI. Springer, 1993.

[106] B.C. Vemuri, S. Rahman, J. Li, Multiresolution adaptive $k$-means algorithm for segmentation of brain MRI. In: Proceedings of the Third International Computer Science Conference (ICSC) on Image Analysis and Computer Graphics, Hong Kong, pp. $347-$ 354, (1995).

[107] L. von Bertalanffy, General Systems Theory, Braziller, New York, 1974. 
[108] C. De Wagter, Optimization of simulated two-dimensional temperature distributions induced by multiple electromagnetic applicators, IEEE Transactions on Microwave Theory and Techniques 34 (1986) 589-596.

[109] J. Warga, Optimal Control of Differential and Functional Equations. Academic Press, New York, 1972.

[110] G.M. Weinberg, An Introduction to General System Theory. Wiley, New York, 1975.

[111] S. Welch, S.S. Gebhart, R.N. Bergman, L.S. Phillips, Minimal model analysis of intravenous glucose tolerance test-derived insulin sensitivity in diabetic subjects, Journal of Clinical Endocrinology Metabolism 71 (1990) 1508-1518.

[112] W.H. Wolberg, W.N. Street, D.M. Heisey, O.L. Mangasarian, Computerized breast cancer diagnosis and prognosis from fineneedle aspirates, Archives of Surgery 130 (1995) 511-516.

[113] P. Wust, J. Nadobny, R. Felix, P. Deuflhard, A. Louis, W. John, Strategies for optimized application of annular-phased-array systems in clinical hyperthermia, International Journal of Hyperthermia 7 (1991) 157-173.

[114] A.W. Wymore, Systems Engineering Methodology for Interdisciplinary Teams. Wiley, New York, 1976.

[115] L. Zadeh, C.A. Desoer, Linear Systems Theory. McGraw-Hill, New York, 1963.

[116] L.H. Zetterberg, Estimation of parameters for a linear difference equation with application to EEG analysis, Mathematical Bioscience 5 (1979) 227-275. 